\title{
Principal Eigenvalues for Isaacs Operators with Neumann Boundary Conditions
}

\author{
Stefania Patrizi
}

\begin{abstract}
In this paper we show the existence of two principal eigenvalues associated to general non-convex fully nonlinear elliptic operators with Neumann boundary conditions in a bounded $C^{2}$ domain. We study these objects and we establish some of their basic properties. Finally, Lipschitz regularity, uniqueness and existence results for the solution of the Neumann problem are given.

Mathematics Subject Classification (2000). 35J60, 35J65, 35P30.

Keywords. Fully nonlinear uniformly elliptic operators, Isaacs operators, Neumann boundary value problems, maximum principle, principal eigenvalues, viscosity solutions.
\end{abstract}

\section{Introduction}

A self-adjoint uniformly elliptic linear operator in a bounded domain possesses a countable set of real eigenvalues, the least of them, called first or principal eigenvalue, can be characterized as the minimum of the associated Rayleigh quotient, see e.g. [14]. This characterization is possible only for operators which are variational. In their famous work [6], Berestycki, Nirenberg and Varadhan defined the principal eigenvalue $\lambda_{1}$ of a general uniformly elliptic linear operator

$$
L[u]=-\operatorname{tr}\left(A(x) D^{2} u\right)+b(x) \cdot D u+c(x) u,
$$

in a bounded domain $\Omega$, as the supremum of those $\lambda$ for which there exists a positive supersolution of $L[u]=\lambda u$. In that paper, they showed that $\lambda_{1}$ is the least eigenvalue of $L$, i.e., for any eigenvalue $\lambda \neq \lambda_{1}, \operatorname{Re}(\lambda)>\lambda_{1}$; moreover $\lambda_{1}$ can be characterized as the supremum of those $\lambda$ for which the operator $L-\lambda I$ satisfies the maximum principle, i.e., for any $\lambda<\lambda_{1}$, if $u$ is a subsolution of $L[u]-\lambda u=0$ and $u \leq 0$ on $\partial \Omega$ then $u \leq 0$ in $\Omega$. Furthermore, they established several other properties of the first eigenvalue, such as simplicity and stability. 
In view of its relation with the maximum and the comparison principles, the concept of principal eigenvalue has been extended to nonlinear operators to study the associated boundary value problems. That has been done for the variational operators, such as the p-Laplacian, through the method of minimization of the so called nonlinear Rayleigh quotient, see e.g. [2] and [20]. An important step in the study of the eigenvalue problem for general nonlinear operators was made by Lions in [21]. In that paper, using probabilistic and analytical methods, he showed the existence of principal eigenvalues for the uniformly elliptic Hamilton-JacobiBellman operator

$$
F\left(x, u, D u, D^{2} u\right)=\sup _{\alpha \in \mathcal{A}}\left\{-\operatorname{tr}\left(A_{\alpha}(x) D^{2} u\right)+b_{\alpha}(x) \cdot D u+c_{\alpha}(x) u\right\},
$$

which arises in stochastic optimal control, see [3] and [12]. Very recently, many authors, inspired by [6], have developed an eigenvalue theory for fully nonlinear operators which are non-variational. The Pucci's extremal operators $\mathcal{M}_{a, A}\left(D^{2} u\right)$ (see the next section) have been treated by Quaas [26] and Busca, Esteban and Quaas [9]. Related results have been obtained by Birindelli and Demengel in [8] for singular or degenerate elliptic operators, like $|D u|^{\alpha} \mathcal{M}_{a, A}\left(D^{2} u\right), \alpha>-1$, the pLaplacian and some its non-variational generalizations. In [27] Quaas and Sirakov have studied the eigenvalue problem for fully nonlinear elliptic operators which are convex and positively homogenous, like the Hamilton-Jacobi-Bellman one (1.1). In that paper many properties of the principal eigenvalues, including the fact that they are simple and isolated, have been established. Similar results have been obtained by Ishii and Yoshimura [19] for non-convex operators, such as the Isaacs one

$$
F\left(x, u, D u, D^{2} u\right)=\sup _{\alpha \in \mathcal{A}} \inf _{\beta \in \mathcal{B}}\left\{-\operatorname{tr}\left(A_{\alpha, \beta}(x) D^{2} u\right)+b_{\alpha, \beta}(x) \cdot D u+c_{\alpha, \beta}(x) u\right\},
$$

which arises in stochastic differential games, see [12].

All these articles treat Dirichlet boundary conditions.

In this paper we want to develop an eigenvalue theory for a class of fully nonlinear operators with Neumann boundary conditions in a bounded $C^{2}$ domain $\Omega$. Precisely, we consider a uniformly elliptic operator which is positively homogenous of order 1

$$
F[u](x)=F\left(x, u, D u, D^{2} u\right),
$$

for any $u \in C^{2}(\bar{\Omega})$, with some additional assumptions that will be made precise in the next section. This class includes the non-convex Isaacs operator (1.2) if $c_{\alpha, \beta}$ are equi-continuous and $A_{\alpha, \beta}, b_{\alpha, \beta}$ are equi-Lipschitz continuous.

To (1.3) we associate the following boundary condition

$$
B(x, u, D u)=f(x, u)+\langle D u, \vec{n}(x)\rangle=0 \quad x \in \partial \Omega,
$$

where $\vec{n}(x)$ is the exterior normal to the domain $\Omega$ at $x$. Typically $f$ will be

$$
f(x, u)=\gamma(x) u,
$$

with $\gamma(x)$ continuous and non-negative on $\partial \Omega$. 
Following the ideas of [6], we define the principal eigenvalues as

$$
\begin{array}{r}
\bar{\lambda}:=\sup \{\lambda \in \mathbb{R} \mid \exists v>0 \text { on } \bar{\Omega} \text { bounded viscosity supersolution of } \\
\left.F\left(x, v, D v, D^{2} v\right)=\lambda v \text { in } \Omega, B(x, v, D v)=0 \text { on } \partial \Omega\right\}, \\
\underline{\lambda}:=\sup \{\lambda \in \mathbb{R} \mid \exists u<0 \text { on } \bar{\Omega} \text { bounded viscosity subsolution of } \\
\left.F\left(x, u, D u, D^{2} u\right)=\lambda u \text { in } \Omega, B(x, u, D u)=0 \text { on } \partial \Omega\right\} .
\end{array}
$$

One of the scope of this work is to prove that $\bar{\lambda}$ and $\underline{\lambda}$ are "eigenvalues" for $F$ which admit respectively a positive and a negative "eigenfunction". Moreover, we show that $\bar{\lambda}$ (resp., $\underline{\lambda}$ ) can be characterized as the supremum of those $\lambda$ for which the operator $F-\lambda I$ with boundary condition (1.4) satisfies the maximum (resp., minimum) principle. As a consequence, $\bar{\lambda}$ (resp., $\underline{\lambda}$ ) is the least "eigenvalue" to which there correspond "eigenfunctions" positive (resp., negative) somewhere.

Other properties of the principal eigenvalues are established: we show that they are simple, isolated and the only "eigenvalues" to which there correspond "eigenfunctions" which do not change sign in $\Omega$. Finally, we obtain Lipschitz regularity, uniqueness and existence results for viscosity solutions of

$$
\begin{cases}F\left(x, u, D u, D^{2} u\right)=g(x) & \text { in } \quad \Omega \\ B(x, u, D u)=0 & \text { on } \partial \Omega .\end{cases}
$$

In particular, we prove that (1.6) is solvable for any continuous right-hand side if the two principal eigenvalues are positive.

The paper is organized as follows. In the next section we give assumptions and precise the notion of solution adopted. In Section 3 we prove the strong comparison principle between sub and supersolutions of (1.6). This allows us to prove the maximum principle for subsolutions of the Neumann boundary value problem. We first show it under the classical assumption that $F$ be proper, see Theorem 4.1; then we prove in Theorem 4.5 that the operator $F-\lambda I$ with boundary condition (1.4) satisfies the maximum principle for any $\lambda<\bar{\lambda}$. Using the example given in [23] we show that the result of Theorem 4.5 is stronger than that of Theorem 4.1, i.e., that there exist non-proper operators which have positive principal eigenvalue $\bar{\lambda}$, and then for which the maximum principle holds.

In Section 5 we establish a Lipschitz regularity result for viscosity solutions of (1.6). In Section 6 we show some existence and comparison theorems. In Section 7 we establish some of the basic properties of the principal eigenvalues. Finally, in Section 8 we show, through an example, that $\bar{\lambda}$ and $\underline{\lambda}$ may be different.

In [23] the author of this paper has studied the principal eigenvalues of fully nonlinear singular elliptic operators modeled on the p-Laplacian or on $|D u|^{\alpha} \mathcal{M}_{a, A}\left(D^{2} u\right), \alpha>-1$, with the pure Neumann boundary condition. In that paper we have used a different approach since the strong comparison principle between sub and supersolutions is not known. For this reason some questions about the properties of the principal eigenvalues, such as simplicity, were left open. 


\section{Assumptions}

Let $S(N)$ be the space of symmetric matrices on $\mathbb{R}^{N}$, equipped with the usual ordering. We denote by $\mathcal{M}_{a, A}^{+}, \mathcal{M}_{a, A}^{-}: S(N) \rightarrow \mathbb{R}$ the Pucci's extremal operators defined by

$$
\begin{aligned}
& \mathcal{M}_{a, A}^{+}(X)=A \sum_{e_{i}>0} e_{i}+a \sum_{e_{i}<0} e_{i}, \\
& \mathcal{M}_{a, A}^{-}(X)=a \sum_{e_{i}>0} e_{i}+A \sum_{e_{i}<0} e_{i},
\end{aligned}
$$

where $e_{1}, \ldots, e_{N}$ are the eigenvalues of $X$ (see e.g. [10]).

The operator $F$ is supposed to be continuous on $\bar{\Omega} \times \mathbb{R} \times \mathbb{R}^{N} \times S(N)$, moreover we shall make the following assumptions:

(F1) For all $(x, r, p, X) \in \bar{\Omega} \times \mathbb{R} \times \mathbb{R}^{N} \times S(N)$ and $t \geq 0$

$$
F(x, t r, t p, t X)=t F(x, r, p, X) .
$$

(F2) There exist $b, c>0$ such that for $x \in \bar{\Omega}, r, s \in \mathbb{R}, p, q \in \mathbb{R}^{N}, X, Y \in S(N)$

$$
\begin{aligned}
\mathcal{M}_{a, A}^{-}(Y-X)-b|p-q|-c|r-s| & \leq F(x, r, p, X)-F(x, s, q, Y) \\
& \leq \mathcal{M}_{a, A}^{+}(Y-X)+b|p-q|+c|r-s| .
\end{aligned}
$$

(F3) For each $T>0$ there exists a continuous function $\omega_{T}$ with $\omega_{T}(0)=0$, such that if $X, Y \in S(N)$ and $\zeta>0$ satisfy

$$
-3 \zeta\left(\begin{array}{cc}
I & 0 \\
0 & I
\end{array}\right) \leq\left(\begin{array}{cc}
X & 0 \\
0 & -Y
\end{array}\right) \leq 3 \zeta\left(\begin{array}{cc}
I & -I \\
-I & I
\end{array}\right)
$$

and $I$ is the identity matrix in $\mathbb{R}^{N}$, then for all $x, y \in \bar{\Omega}, r \in[-T, T]$, $p \in \mathbb{R}^{N}$

$$
F(y, r, p, Y)-F(x, r, p, X) \leq \omega_{T}\left(\zeta|x-y|^{2}+|x-y|(|p|+1)\right) .
$$

(F4) There exists $C_{1}>0$ such that for all $x, y \in \bar{\Omega}$ and $X \in S(N)$

$$
|F(x, 0,0, X)-F(y, 0,0, X)| \leq C_{1}|x-y|^{\frac{1}{2}}\|X\| \text {. }
$$

Here and in what follows we fix the norm $\|X\|$ in $S(N)$ by setting

$$
\|X\|=\sup \left\{|X \xi|: \xi \in \mathbb{R}^{N},|\xi| \leq 1\right\}=\sup \{|\lambda|: \lambda \text { is an eigenvalue of } X\} .
$$

Remark that (F1) implies that $F(x, 0,0,0) \equiv 0$.

The Isaacs operator (1.2) is continuous and satisfies (F1) and (F2) if $a I \leq$ $A_{\alpha, \beta}(x) \leq A I$ for any $x \in \bar{\Omega},(\alpha, \beta) \in \mathcal{A} \times \mathcal{B}$ and the functions $A_{\alpha, \beta}, b_{\alpha, \beta}, c_{\alpha, \beta}$ are continuous on $\bar{\Omega}$ uniformly in $\alpha$ and $\beta$, where $\mathcal{A}$ and $\mathcal{B}$ are arbitrary index sets. If the matrices $A_{\alpha, \beta}$ are equi-Hölderian of exponent $\frac{1}{2}$, i.e., for some constant $C>0$

$$
\left\|A_{\alpha, \beta}(x)-A_{\alpha, \beta}(y)\right\| \leq C|x-y|^{\frac{1}{2}} \quad \text { for all } \quad x, y \in \bar{\Omega} \quad \text { and } \quad(\alpha, \beta) \in \mathcal{A} \times \mathcal{B},
$$

then $F$ satisfies (F4). Finally, (F3) is satisfied by $F$ if, in addition to the uniform elliptic condition $A_{\alpha, \beta}(x) \geq a I$ and the equi-continuity of $c_{\alpha, \beta}$, the functions $A_{\alpha, \beta}$ 
and $b_{\alpha, \beta}$ are equi-Lipschitz continuous, i.e., there exists $L>0$ such that for all $x, y \in \bar{\Omega}$ and $(\alpha, \beta) \in \mathcal{A} \times \mathcal{B}$

$$
\left\|A_{\alpha, \beta}(x)-A_{\alpha, \beta}(y)\right\| \leq L|x-y|, \quad\left|b_{\alpha, \beta}(x)-b_{\alpha, \beta}(y)\right| \leq L|x-y| .
$$

We assume throughout the paper that $\Omega$ is a bounded domain of $\mathbb{R}^{N}$ of class $C^{2}$. In particular it satisfies the interior sphere condition and the uniform exterior sphere condition, i.e.,

$(\Omega 1)$ For each $x \in \partial \Omega$ there exist $R>0$ and $y \in \Omega$ for which $|x-y|=R$ and $B(y, R) \subset \Omega$.

$(\Omega 2)$ There exists $r>0$ such that $B(x+r \vec{n}(x), r) \cap \Omega=\emptyset$ for any $x \in \partial \Omega$.

From property $(\Omega 2)$ it follows that

$$
\langle\vec{n}(x), y-x\rangle \leq \frac{1}{2 r}|y-x|^{2} \quad \text { for } \quad x \in \partial \Omega \quad \text { and } \quad y \in \bar{\Omega} .
$$

Moreover, the $C^{2}$-regularity of $\Omega$ implies the existence of a neighborhood of $\partial \Omega$ in $\bar{\Omega}$ on which the distance from the boundary

$$
d(x):=\inf \{|x-y|, y \in \partial \Omega\}, \quad x \in \bar{\Omega}
$$

is of class $C^{2}$. We still denote by $d$ a $C^{2}$ extension of the distance function to the whole $\bar{\Omega}$. Without loss of generality we can assume that $|\operatorname{Dd}(x)| \leq 1$ on $\bar{\Omega}$.

On the function $f$ in (1.4) we shall suppose

(f1) $f: \partial \Omega \times \mathbb{R} \rightarrow \mathbb{R}$ is continuous.

(f2) For all $(x, r) \in \partial \Omega \times \mathbb{R}$ and $t \geq 0$

$$
f(x, t r)=t f(x, r) .
$$

For the existence results we will assume in addition

(f3) For all $x \in \partial \Omega r \rightarrow f(x, r)$ is non-decreasing on $\mathbb{R}$.

Clearly, $f(x, u)=\gamma(x) u$ with $\gamma(x) \geq 0$ and continuous on $\partial \Omega$, satisfies all the three hypothesis.

In this paper we adopt the notion of viscosity solution. We denote by $U S C(\bar{\Omega})$ the set of upper semicontinuous functions on $\bar{\Omega}$ and by $L S C(\bar{\Omega})$ the set of lower semicontinuous functions on $\bar{\Omega}$. Given $g: \bar{\Omega} \rightarrow \mathbb{R}$, we recall that a function $u \in$ $U S C(\bar{\Omega})$ (resp., $u \in L S C(\bar{\Omega})$ ) is called viscosity subsolution (resp., supersolution) of

$$
\begin{cases}F\left(x, u, D u, D^{2} u\right)=g(x) & \text { in } \quad \Omega \\ B(x, u, D u)=0 & \text { on } \quad \partial \Omega .\end{cases}
$$

if the following conditions hold

(i) For every $x_{0} \in \Omega$ for all $\varphi \in C^{2}(\bar{\Omega})$, such that $u-\varphi$ has a local maximum (resp., minimum) on $x_{0}$ then

$$
F\left(x_{0}, u\left(x_{0}\right), D \varphi\left(x_{0}\right), D^{2} \varphi\left(x_{0}\right)\right) \leq(\text { resp. }, \geq) g\left(x_{0}\right) .
$$


(ii) For every $x_{0} \in \partial \Omega$ for all $\varphi \in C^{2}(\bar{\Omega})$, such that $u-\varphi$ has a local maximum (resp., minimum) on $x_{0}$ then

$$
\begin{aligned}
& \left(F\left(x_{0}, u\left(x_{0}\right), D \varphi\left(x_{0}\right), D^{2} \varphi\left(x_{0}\right)\right)-g\left(x_{0}\right)\right) \wedge B\left(x_{0}, u\left(x_{0}\right), D \varphi\left(x_{0}\right)\right) \leq 0 \\
& (\text { resp., } \\
& \left.\left(F\left(x_{0}, u\left(x_{0}\right), D \varphi\left(x_{0}\right), D^{2} \varphi\left(x_{0}\right)\right)-g\left(x_{0}\right)\right) \vee B\left(x_{0}, u\left(x_{0}\right), D \varphi\left(x_{0}\right)\right) \geq 0 .\right)
\end{aligned}
$$

A viscosity solution is a continuous function which is both a subsolution and a supersolution.

In the above definition the test functions can be substituted by the elements of the semi-jets $\bar{J}^{2,+} u\left(x_{0}\right)$ when $u$ is a subsolution and $\bar{J}^{2,-} u\left(x_{0}\right)$ when $u$ is a supersolution. For a detailed presentation of the theory of viscosity solutions we refer the reader to e.g. [11].

One of the motivation for these relaxed boundary conditions is the stability under uniform convergence. Actually, if the operator $F$ satisfies (F2) and the domain $\Omega$ the exterior sphere condition, viscosity subsolutions (resp., supersolutions) satisfy in the viscosity sense $B(x, u(x), D u(x)) \leq($ resp. $\geq) 0$ for any $x \in \partial \Omega$, as shown in the following proposition due to Hitoshi Ishii, [17], whose proof is given for the reader's convenience.

Proposition 2.1. Suppose that $\Omega$ satisfies the exterior sphere condition. If there exists $b>0$ such that for $x \in \bar{\Omega}, r \in \mathbb{R}, p, q \in \mathbb{R}^{N}, X, Y \in \mathrm{S}(\mathrm{N})$

$$
F(x, r, p, X)-F(x, r, q, Y) \geq \mathcal{M}_{a, A}^{-}(Y-X)-b|p-q|,
$$

and $u$ is a viscosity subsolution of (2.2) then $u$ satisfies in the viscosity sense

$$
B\left(x_{0}, u\left(x_{0}\right), D u\left(x_{0}\right)\right) \leq 0,
$$

for any $x_{0} \in \partial \Omega$. If

$$
F(x, r, p, X)-F(x, r, q, Y) \leq \mathcal{M}_{a, A}^{+}(Y-X)+b|p-q|,
$$

and $u$ is a viscosity supersolution of (2.2) then $u$ satisfies in the viscosity sense

$$
B\left(x_{0}, u\left(x_{0}\right), D u\left(x_{0}\right)\right) \geq 0,
$$

for any $x_{0} \in \partial \Omega$.

Proof. We show the proposition for subsolutions. Set

$$
g(t)=-K t^{2}+\epsilon t \quad \forall t \in \mathbb{R},
$$

where $K \gg 1$ and $0<\epsilon \ll 1$. Observe that $g(0)=0, g^{\prime}(0)=\epsilon, g^{\prime \prime}(0)=-2 K$, and

$$
0<t<\frac{\epsilon}{K} \quad \Longrightarrow \quad g(t)>0 \text {. }
$$

Let $\varphi \in C^{2}(\bar{\Omega})$ and $x_{0} \in \partial \Omega$. Assume that $u-\varphi$ attains a maximum at $x_{0}$. We need to prove that $f\left(x_{0}, u\left(x_{0}\right)\right)+\left\langle\vec{n}\left(x_{0}\right), D \varphi\left(x_{0}\right)\right\rangle \leq 0$.

Let $y_{0} \in \mathbb{R}^{N}$ and $R>0$ satisfy

$$
B\left(y_{0}, R\right) \cap \bar{\Omega}=\left\{x_{0}\right\} .
$$


We may assume by translation that $y_{0}=0$. We set

$$
\psi(x)=g(|x|-R) \quad \forall x \in \mathbb{R}^{N} .
$$

Note that $\psi\left(x_{0}\right)=g(0)=0$,

$$
\begin{aligned}
D \psi\left(x_{0}\right) & =g^{\prime}(0) \frac{x_{0}}{\left|x_{0}\right|}=\epsilon e_{0}, \quad \text { where } e_{0}=\frac{x_{0}}{\left|x_{0}\right|}, \\
\vec{n}\left(x_{0}\right) \cdot D \psi\left(x_{0}\right) & =-e_{0} \cdot \epsilon e_{0}=-\epsilon, \\
D^{2} \psi\left(x_{0}\right) & =g^{\prime \prime}(0) e_{0} \otimes e_{0}+\frac{g^{\prime}(0)}{\left|x_{0}\right|}\left(I-e_{0} \otimes e_{0}\right) \\
& =-2 K e_{0} \otimes e_{0}+\frac{\epsilon}{R}\left(I-e_{0} \otimes e_{0}\right), \\
\mathcal{M}_{a, A}^{-}\left(-D^{2} \psi\left(x_{0}\right)\right) & =-\frac{\epsilon(N-1) A}{R}+2 K a, \\
R & <|x|<R+\frac{\epsilon}{K} \Longrightarrow \psi(x)>0 .
\end{aligned}
$$

Moreover we observe that $u-\varphi-\psi$ attains a local maximum at $x_{0}$. Remark that

$$
\begin{aligned}
& F\left(x_{0}, u\left(x_{0}\right), D \varphi\left(x_{0}\right)+D \psi\left(x_{0}\right), D^{2} \varphi\left(x_{0}\right)+D^{2} \psi\left(x_{0}\right)\right) \\
& \quad \geq F\left(x_{0}, u\left(x_{0}\right), D \varphi\left(x_{0}\right), D^{2} \varphi\left(x_{0}\right)\right)-b\left|D \psi\left(x_{0}\right)\right|+\mathcal{M}_{a, A}^{-}\left(-D^{2} \psi\left(x_{0}\right)\right) \\
& \quad \geq F\left(x_{0}, u\left(x_{0}\right), D \varphi\left(x_{0}\right), D^{2} \varphi\left(x_{0}\right)\right)-b \epsilon-\frac{\epsilon(N-1) A}{R}+2 K a .
\end{aligned}
$$

We fix $K \gg 1$ so that for any $0<\epsilon<1$

$$
F\left(x_{0}, u\left(x_{0}\right), D \varphi\left(x_{0}\right), D^{2} \varphi\left(x_{0}\right)\right)-b \epsilon-\frac{\epsilon(N-1) A}{R}+2 K a>g\left(x_{0}\right) .
$$

Then, by definition of subsolution we get that

$0 \geq f\left(x_{0}, u\left(x_{0}\right)\right)+\vec{n}\left(x_{0}\right) \cdot\left(D \varphi\left(x_{0}\right)+D \psi\left(x_{0}\right)\right)=f\left(x_{0}, u\left(x_{0}\right)\right)+\vec{n}\left(x_{0}\right) \cdot D \varphi\left(x_{0}\right)-\epsilon$, from which we obtain

$$
f\left(x_{0}, u\left(x_{0}\right)\right)+\vec{n}\left(x_{0}\right) \cdot D \varphi\left(x_{0}\right) \leq 0,
$$

as desired

\section{The strong comparison principle}

The strong comparison principle is the key ingredient in the development of our theory.

Theorem 3.1. Assume that (F2), (F3), (f1) hold and that $g$ is continuous on $\bar{\Omega}$. Let $u \in U S C(\bar{\Omega})$ and $v \in L S C(\bar{\Omega})$ be respectively a sub and a supersolution of

$$
\begin{cases}F\left(x, u, D u, D^{2} u\right)=g(x) & \text { in } \Omega \\ B(x, u, D u)=0 & \text { on } \partial \Omega .\end{cases}
$$

If $u \leq v$ on $\bar{\Omega}$ then either $u<v$ on $\bar{\Omega}$ or $u \equiv v$ on $\bar{\Omega}$. 
Let us recall that for the sub and the supersolutions of the Dirichlet problem the following theorem holds, see [19].

Theorem 3.2. Assume that (F2), (F3) hold and that $g$ is continuous on $\Omega$. Let $u \in U S C(\Omega)$ and $v \in L S C(\Omega)$ be respectively a sub and a supersolution of

$$
F\left(x, u, D u, D^{2} u\right)=g(x) .
$$

If $u \leq v$ in $\Omega$ then either $u<v$ in $\Omega$ or $u \equiv v$ in $\Omega$.

Proof of Theorem 3.1. Assume $u \not \equiv v$, then by Theorem $3.2 u<v$ in $\Omega$. Suppose by contradiction that there exists a point $x_{0} \in \partial \Omega$ on which $u\left(x_{0}\right)=v\left(x_{0}\right)$.

The interior sphere condition $(\Omega 1)$ implies that there exist $R>0$ and $y_{0} \in \Omega$ such that the ball centered in $y_{0}$ and of radius $R, B_{1}$, is contained in $\Omega$ and $x_{0} \in \partial B_{1}$. Let for $k>2 / R^{2}$ and $x \in \bar{\Omega}$

$$
w(x):=e^{-k R^{2}}-e^{-k\left|x-y_{0}\right|^{2}} .
$$

This function has the following properties

$$
\begin{aligned}
& w(x)<0 \quad \text { in } B_{1}, \\
& w(x)=0 \quad \text { on } \partial B_{1}, \\
& w(x)>0 \quad \text { outside } \bar{B}_{1} .
\end{aligned}
$$

Let $B_{2}$ be the ball of center $y_{0}$ and radius $\frac{R}{2}$ and $-m:=\max _{\bar{B}_{2}}(u-v)<0$. Choose $\sigma>0$ so small that

$$
\sigma \inf _{\bar{B}_{2}} w \geq-\frac{m}{2} .
$$

Let us define for $j \in \mathbb{N}$ the functions

$$
\phi(x, y):=\frac{j}{2}|x-y|^{2}+\frac{\sigma}{2}(w(x)+w(y))-f\left(x_{0}, u\left(x_{0}\right)\right)\left\langle\vec{n}\left(x_{0}\right), x-y\right\rangle,
$$

and

$$
\psi(x, y):=u(x)-v(y)-\phi(x, y) .
$$

Let $\left(x_{j}, y_{j}\right) \in \bar{\Omega}^{2}$ be a maximum point of $\psi$ in $\bar{\Omega}^{2}$. We have

$$
\begin{aligned}
0= & u\left(x_{0}\right)-v\left(x_{0}\right)-\sigma w\left(x_{0}\right) \\
\leq & u\left(x_{j}\right)-v\left(y_{j}\right)-\frac{j}{2}\left|x_{j}-y_{j}\right|^{2}-\frac{\sigma}{2}\left(w\left(x_{j}\right)+w\left(y_{j}\right)\right) \\
& +f\left(x_{0}, u\left(x_{0}\right)\right)\left\langle\vec{n}\left(x_{0}\right), x_{j}-y_{j}\right\rangle,
\end{aligned}
$$

from which we can see that $\left|x_{j}-y_{j}\right| \rightarrow 0$ as $j \rightarrow+\infty$. Up to subsequence, $x_{j}$ and $y_{j}$ converge to some $\bar{z} \in \bar{\Omega}$. Standard arguments show that

$$
\lim _{j \rightarrow+\infty} \frac{j}{2}\left|x_{j}-y_{j}\right|^{2}=0, \quad \lim _{j \rightarrow+\infty} u\left(x_{j}\right) \rightarrow u(\bar{z}) \quad \text { and } \quad \lim _{j \rightarrow+\infty} v\left(y_{j}\right) \rightarrow v(\bar{z}) .
$$

Passing to the limit in (3.2) we get

$$
\sigma w(\bar{z}) \leq u(\bar{z})-v(\bar{z}) \leq 0
$$


which implies that the limit point $\bar{z}$ belongs to $\bar{B}_{1}$. Furthermore, since $u(\bar{z})-v(\bar{z})-$ $\sigma w(\bar{z}) \geq 0$, it cannot belong to $\bar{B}_{2}$, indeed by (3.1) we have $u(x)-v(x)-\sigma w(x) \leq$ $-\frac{m}{2}<0$, for any $x \in \bar{B}_{2}$. In conclusion

$$
\frac{R}{2}<\left|\bar{z}-y_{0}\right| \leq R
$$

Computing the derivatives of $\phi$ we get

$$
\begin{aligned}
& D_{x} \phi(x, y)=j(x-y)+\sigma k e^{-k\left|x-y_{0}\right|^{2}}\left(x-y_{0}\right)-f\left(x_{0}, u\left(x_{0}\right)\right) \vec{n}\left(x_{0}\right), \\
& D_{y} \phi(x, y)=-j(x-y)+\sigma k e^{-k\left|y-y_{0}\right|^{2}}\left(y-y_{0}\right)+f\left(x_{0}, u\left(x_{0}\right)\right) \vec{n}\left(x_{0}\right) .
\end{aligned}
$$

If $x_{j} \in \partial \Omega$ then $\bar{z}=x_{0}$ and using (2.1) we have

$$
\begin{aligned}
B\left(x_{j}, u\left(x_{j}\right), D_{x} \phi\left(x_{j}, y_{j}\right)\right) \geq & f\left(x_{j}, u\left(x_{j}\right)\right)-f\left(x_{0}, u\left(x_{0}\right)\right)\left\langle\vec{n}\left(x_{0}\right), \vec{n}\left(x_{j}\right)\right\rangle \\
& -\frac{j}{2 r}\left|x_{j}-y_{j}\right|^{2}+\sigma k e^{-k\left|x_{j}-y_{0}\right|^{2}}\left\langle x_{j}-y_{0}, \vec{n}\left(x_{j}\right)\right\rangle>0
\end{aligned}
$$

for large $j$, since the last term goes to $\sigma k e^{-k R^{2}} R$ as $j \rightarrow+\infty$, being $\vec{n}\left(x_{0}\right)=$ $\frac{x_{0}-y_{0}}{R}$. Similarly if $y_{j} \in \partial \Omega$ then $\bar{z}=x_{0}$ and $u\left(x_{0}\right)=v\left(x_{0}\right)$ so that

$$
\begin{aligned}
B\left(y_{j}, v\left(y_{j}\right),-D_{y} \phi\left(x_{j}, y_{j}\right)\right) \leq & f\left(y_{j}, v\left(y_{j}\right)\right)-f\left(x_{0}, u\left(x_{0}\right)\right)\left\langle\vec{n}\left(x_{0}\right), \vec{n}\left(y_{j}\right)\right\rangle \\
& +\frac{j}{2 r}\left|x_{j}-y_{j}\right|^{2}-\sigma k e^{-k\left|y_{j}-y_{0}\right|^{2}}\left\langle y_{j}-y_{0}, \vec{n}\left(y_{j}\right)\right\rangle<0
\end{aligned}
$$

for large $j$. Then $x_{j}$ and $y_{j}$ are internal points and

$$
\begin{gathered}
F\left(x_{j}, u\left(x_{j}\right), D_{x} \phi\left(x_{j}, y_{j}\right), X\right) \leq g\left(x_{j}\right) \quad \text { if } \quad\left(D_{x} \phi\left(x_{j}, y_{j}\right), X\right) \in \bar{J}^{2,+} u\left(x_{j}\right), \\
F\left(y_{j}, v\left(y_{j}\right),-D_{y} \phi\left(x_{j}, y_{j}\right), Y\right) \geq g\left(y_{j}\right) \quad \text { if } \quad\left(-D_{y} \phi\left(x_{j}, y_{j}\right), Y\right) \in \bar{J}^{2,-} v\left(y_{j}\right) .
\end{gathered}
$$

Then the previous relations hold for $\left(x_{j}, y_{j}\right) \in \bar{\Omega}^{2}$, provided $j$ is large.

Since $\left(x_{j}, y_{j}\right)$ is a local maximum point of $\psi(x, y)=\left(u(x)-\frac{\sigma}{2} w(x)\right)-$ $\left(v(y)+\frac{\sigma}{2} w(y)\right)-\frac{j}{2}|x-y|^{2}+f\left(x_{0}, u\left(x_{0}\right)\right)\left\langle\vec{n}\left(x_{0}\right), x-y\right\rangle$ in $\bar{\Omega}^{2}$, applying Theorem 3.2 of [11] there exist $X_{j}, Y_{j} \in S(N)$ such that $\left(D_{x} \phi\left(x_{j}, y_{j}\right), X_{j}\right) \in \bar{J}^{2,+} u\left(x_{j}\right)$, $\left(-D_{y} \phi\left(x_{j}, y_{j}\right), Y_{j}\right) \in \bar{J}^{2,-} v\left(y_{j}\right)$ and

$-3 j\left(\begin{array}{cc}I & 0 \\ 0 & I\end{array}\right) \leq\left(\begin{array}{cc}X_{j}-\frac{\sigma}{2} D^{2} w\left(x_{j}\right) & 0 \\ 0 & -\left(Y_{j}+\frac{\sigma}{2} D^{2} w\left(y_{j}\right)\right)\end{array}\right) \leq 3 j\left(\begin{array}{cc}I & -I \\ -I & I\end{array}\right)$.

The hessian matrix of $w(x)$ is

$$
D^{2} w(x)=2 k e^{-k\left|x-y_{0}\right|^{2}} I-4 k^{2} e^{-k\left|x-y_{0}\right|^{2}}\left(x-y_{0}\right) \otimes\left(x-y_{0}\right) .
$$

Its eigenvalues are $2 k e^{-k\left|x-y_{0}\right|^{2}}$ with multiplicity $N-1$ and $2 k e^{-k\left|x-y_{0}\right|^{2}}(1-2 k \mid x-$ $\left.\left.y_{0}\right|^{2}\right)$ with multiplicity 1 . In the annulus $B_{1} \backslash \bar{B}_{2}$ we have $2 k e^{-k\left|x-y_{0}\right|^{2}}(1-2 k \mid x-$ $\left.\left.y_{0}\right|^{2}\right) \leq 2 k e^{-k\left|x-y_{0}\right|^{2}}\left(1-k \frac{R^{2}}{2}\right)<0$ since $k>\frac{2}{R^{2}}$. 
Using the fact that $u$ and $v$ are respectively sub and supersolution and the properties of the operator $F$ we have

$$
\begin{aligned}
g\left(y_{j}\right) \leq & F\left(y_{j}, v\left(y_{j}\right),-D_{y} \phi, Y_{j}\right) \\
\leq & F\left(y_{j}, v\left(y_{j}\right),-D_{y} \phi, Y_{j}+\frac{\sigma}{2} D^{2} w\left(y_{j}\right)\right)+\frac{\sigma}{2} \mathcal{M}_{a, A}^{+}\left(D^{2} w\left(y_{j}\right)\right) \\
\leq & F\left(x_{j}, v\left(y_{j}\right),-D_{y} \phi, X_{j}-\frac{\sigma}{2} D^{2} w\left(x_{j}\right)\right)+\omega_{T}\left(o_{j}\right)+\frac{\sigma}{2} \mathcal{M}_{a, A}^{+}\left(D^{2} w\left(y_{j}\right)\right) \\
\leq & F\left(x_{j}, u\left(x_{j}\right), D_{x} \phi, X_{j}\right)+\omega_{T}\left(o_{j}\right)+\frac{\sigma}{2} \mathcal{M}_{a, A}^{+}\left(D^{2} w\left(x_{j}\right)\right) \\
& +\frac{\sigma}{2} \mathcal{M}_{a, A}^{+}\left(D^{2} w\left(y_{j}\right)\right)+b \frac{\sigma}{2}\left|D w\left(x_{j}\right)\right|+b \frac{\sigma}{2}\left|D w\left(y_{j}\right)\right|+c\left|u\left(x_{j}\right)-v\left(y_{j}\right)\right| \\
\leq & g\left(x_{j}\right)+\omega_{T}\left(o_{j}\right)+\frac{\sigma}{2} \mathcal{M}_{a, A}^{+}\left(D^{2} w\left(x_{j}\right)\right)+\frac{\sigma}{2} \mathcal{M}_{a, A}^{+}\left(D^{2} w\left(y_{j}\right)\right)+b \frac{\sigma}{2}\left|D w\left(x_{j}\right)\right| \\
& +b \frac{\sigma}{2}\left|D w\left(y_{j}\right)\right|+c\left|u\left(x_{j}\right)-v\left(y_{j}\right)\right|,
\end{aligned}
$$

where $o_{j}=j\left|x_{j}-y_{j}\right|^{2}+\left|x_{j}-y_{j}\right|\left(\left|D_{y} \phi\right|+1\right) \rightarrow 0$ as $j \rightarrow+\infty$. Then

$$
\begin{aligned}
g\left(y_{j}\right) \leq & g\left(x_{j}\right)+A(N-1) \sigma k e^{-k\left|x_{j}-y_{0}\right|^{2}}+a \sigma k e^{-k\left|x_{j}-y_{0}\right|^{2}}\left(1-2 k\left|x_{j}-y_{0}\right|^{2}\right) \\
& +A(N-1) \sigma k e^{-k\left|y_{j}-y_{0}\right|^{2}}+a \sigma k e^{-k\left|y_{j}-y_{0}\right|^{2}}\left(1-2 k\left|y_{j}-y_{0}\right|^{2}\right) \\
& +k \sigma e^{-k\left|x_{j}-y_{0}\right|^{2}} b\left|x_{j}-y_{0}\right|+k \sigma e^{-k\left|y_{j}-y_{0}\right|^{2}} b\left|y_{j}-y_{0}\right| \\
& +c\left|u\left(x_{j}\right)-v\left(y_{j}\right)\right|+\omega_{T}\left(o_{j}\right) .
\end{aligned}
$$

Passing to the limit as $j \rightarrow+\infty$ we get

$$
2 \sigma e^{-k\left|\bar{z}-y_{0}\right|^{2}}\left\{-2 a k^{2}\left|\bar{z}-y_{0}\right|^{2}+\left[A(N-1)+a+b\left|\bar{z}-y_{0}\right|\right] k\right\}+c|u(\bar{z})-v(\bar{z})| \geq 0 .
$$

Using (3.3) and the fact that $\frac{R}{2}<\left|\bar{z}-y_{0}\right| \leq R$, we have

$$
\begin{aligned}
0 & \leq 2 \sigma e^{-k\left|\bar{z}-y_{0}\right|^{2}}\left\{-2 a k^{2}\left|\bar{z}-y_{0}\right|^{2}+\left[A(N-1)+a+b\left|\bar{z}-y_{0}\right|\right] k\right\}+c|u(\bar{z})-v(\bar{z})| \\
& \leq 2 \sigma e^{-k\left|\bar{z}-y_{0}\right|^{2}}\left\{-a k^{2} \frac{R^{2}}{2}+[A(N-1)+a+b R] k\right\}+\sigma c\left(e^{-k\left|\bar{z}-y_{0}\right|^{2}}-e^{-k R^{2}}\right) \\
& \leq \sigma e^{-k\left|\bar{z}-y_{0}\right|^{2}}\left\{-a k^{2} R^{2}+2[A(N-1)+a+b R] k+c\right\} .
\end{aligned}
$$

If we fix $k>2 / R^{2}$ so large that

$$
-a k^{2} R^{2}+2[A(N-1)+a+b R] k+c<0,
$$

we obtain a contradiction, then $u<v$ on $\bar{\Omega}$.

Remark 3.3. In Theorem 3.1 the domain $\Omega$ may be unbounded. In that case, in the proof of the theorem it suffices to maximize $\psi(x, y)$ on the compact set $\left(\bar{B}\left(y_{0}, 2 R\right) \cap \bar{\Omega}\right)^{2}$, instead of the whole $\bar{\Omega}$.

A consequence of Theorem 3.1 are the following strong maximum and minimum principles. 
Corollary 3.4. Assume the hypothesis of Theorem 3.1. If $f(x, 0) \leq 0$ for any $x \in \partial \Omega$ and $v \in L S C(\bar{\Omega})$ is a non-negative viscosity supersolution of

$$
\begin{cases}F\left(x, v, D v, D^{2} v\right)=0 & \text { in } \Omega \\ B(x, v, D v)=0 & \text { on } \partial \Omega,\end{cases}
$$

then either $v \equiv 0$ or $v>0$ on $\bar{\Omega}$. If $f(x, 0) \geq 0$ for any $x \in \partial \Omega$ and $u \in U S C(\bar{\Omega})$ is a non-positive viscosity subsolution of (3.4) then either $u \equiv 0$ or $u<0$ on $\bar{\Omega}$.

Proof. If $f(x, 0) \leq 0$ for any $x \in \partial \Omega$ then $u \equiv 0$ is a subsolution of (3.4). The thesis follows applying Theorem 3.1.

\section{The maximum principle and the principal eigenvalues}

We say that $F$ with boundary condition (1.4) satisfies the maximum principle, if whenever $u \in U S C(\bar{\Omega})$ is a viscosity subsolution of

$$
\begin{cases}F\left(x, u, D u, D^{2} u\right)=0 & \text { in } \Omega \\ B(x, u, D u)=0 & \text { on } \partial \Omega,\end{cases}
$$

then $u \leq 0$ on $\bar{\Omega}$. We first prove that the maximum principle holds if $F$ is proper, i.e., if $r \rightarrow F(x, r, p, M)$ is non-decreasing. Observe that we do not require the stronger condition $F(x, r, p, X)-\sigma r$ non-decreasing in $r$ for some $\sigma>0$, in which case the comparison principle holds (see [11] Theorem 7.5) and implies the maximum principle if $u \equiv 0$ is a supersolution.

Successively, we show that the operator $F-\lambda I$ with boundary condition (1.4) satisfies the maximum principle for any $\lambda<\bar{\lambda}$. To prove that the two results do not coincide, we construct a class of operators which are not proper but that have positive principal eigenvalue $\bar{\lambda}$, hence for which the maximum principle holds.

\subsection{The case $\mathbf{F}$ proper}

Theorem 4.1. Assume that (F2), (F3), (f1) and (f3) hold, that $r \rightarrow F(x, r, p, M)$ is non-decreasing on $\mathbb{R}$ for all $(x, p, M) \in \bar{\Omega} \times \mathbb{R}^{N} \times S(N), F(x, 0,0,0) \geq 0$ for all $x \in \Omega, f(x, 0) \geq 0$ for all $x \in \partial \Omega$ and

$$
\max _{x \in \partial \Omega} f(x, r) \vee \max _{x \in \bar{\Omega}} F(x, r, 0,0)>0 \quad \text { for any } \quad r>0 .
$$

If $u \in U S C(\bar{\Omega})$ is a viscosity subsolution of

$$
\begin{cases}F\left(x, u, D u, D^{2} u\right)=0 & \text { in } \quad \Omega \\ B(x, u, D u)=0 & \text { on } \partial \Omega,\end{cases}
$$

then $u \leq 0$ on $\bar{\Omega}$. 
Proof. Let $u$ be a subsolution of (4.2). First let us suppose $u \equiv k=$ const. By definition of subsolution and Proposition 2.1

$$
F(x, k, 0,0) \leq 0, \quad \text { for any } \quad x \in \Omega
$$

and

$$
B(x, k, 0)=f(x, k) \leq 0 \quad \text { for any } \quad x \in \partial \Omega .
$$

Then the hypothesis (4.1) implies $k \leq 0$.

Now we assume that $u$ is not a constant. We argue by contradiction; suppose that $\max _{\bar{\Omega}} u=u\left(x_{0}\right)>0$, for some $x_{0} \in \bar{\Omega}$. Define $\widetilde{u}(x):=u(x)-u\left(x_{0}\right)$. Since $r \rightarrow$ $F(x, r, p, M)$ and $r \rightarrow f(x, r)$ are non-decreasing, $\widetilde{u}$ is a non-positive subsolution of (4.2). The properties $F(x, 0,0,0) \geq 0$ and $f(x, 0) \geq 0$ imply that $v \equiv 0$ is a supersolution of (4.2). Then it follows from Theorem 3.1 that either $u \equiv u\left(x_{0}\right)$ or $u<u\left(x_{0}\right)$ on $\bar{\Omega}$. In both cases we get a contradiction.

Remark 4.2. Under the assumptions of Theorem 4.1, but now with $F(x, 0,0,0) \leq 0$ for all $x \in \Omega, f(x, 0) \leq 0$ for all $x \in \partial \Omega$ and $\min _{x \in \partial \Omega} f(x, r) \vee \min _{x \in \bar{\Omega}} F(x, r, 0,0)<$ 0 for any $r<0$, we can prove the minimum principle, i.e., if $u \in L S C(\bar{\Omega})$ is a viscosity supersolution of (4.2) then $u \geq 0$ on $\bar{\Omega}$.

Remark 4.3. If $F$ does not depend on $r$ and $f \equiv 0$ a counterexample to the validity of the maximum principle is given by the positive constants.

4.2. The maximum principle for $\lambda<\bar{\lambda}$

We set

$$
\begin{array}{r}
\bar{E}:=\{\lambda \in \mathbb{R} \mid \exists v>0 \text { on } \bar{\Omega} \text { bounded viscosity supersolution of } \\
\left.F\left(x, v, D v, D^{2} v\right)=\lambda v \text { in } \Omega, B(x, v, D v)=0 \text { on } \partial \Omega\right\}, \\
\underline{E}:=\{\lambda \in \mathbb{R} \mid \exists u<0 \text { on } \bar{\Omega} \text { bounded viscosity subsolution of } \\
\left.F\left(x, u, D u, D^{2} u\right)=\lambda u \text { in } \Omega, B(x, u, D u)=0 \text { on } \partial \Omega\right\} .
\end{array}
$$

The set $\bar{E}$ is not empty, indeed the function $v(x)=e^{-|f(\cdot, 1)|_{\infty} d(x)}$ satisfies

$$
\begin{aligned}
F\left(x, v, D v, D^{2} v\right)-\lambda v \geq & e^{-|f(\cdot, 1)|_{\infty} d(x)}\left\{-\mathcal{M}_{a, A}^{+}\left(|f(\cdot, 1)|_{\infty}^{2} \operatorname{Dd}(x) \otimes \operatorname{Dd}(x)\right.\right. \\
& \left.\left.-|f(\cdot, 1)|_{\infty} D^{2} d(x)\right)-b|f(\cdot, 1)|_{\infty}-c-\lambda\right\} \geq 0,
\end{aligned}
$$

in $\Omega$, for $\lambda$ small enough, and

$$
B(x, v, D v)=f(x, 1)+|f(x, 1)|_{\infty} \geq 0,
$$

on $\partial \Omega$. As a consequence $\bar{\lambda}=\sup \bar{E}$ is well defined. Similarly we can prove that $\underline{E}$ is not empty. We shall show that $\bar{\lambda}$ and $\underline{\lambda}$ are finite.

We want to remark that since in the sequel we will assume (f2), which implies $f(x, 0)=0$ for any $x \in \partial \Omega$, by Corollary 3.4 any non-negative supersolution (resp., non-positive subsolution) of $F\left(x, v, D v, D^{2} v\right)=0$ in $\Omega, B(x, v, D v)=0$ on $\partial \Omega$ which is non-zero will be positive (resp., negative) in all $\bar{\Omega}$. 
Theorem 4.4. Assume that (F1)-(F3), (f1) and (f2) hold. Let $u \in U S C(\bar{\Omega})$ and $v \in L S C(\bar{\Omega})$ be respectively sub and supersolution of

$$
\begin{cases}F\left(x, u, D u, D^{2} u\right)=0 & \text { in } \quad \Omega \\ B(x, u, D u)=0 & \text { on } \partial \Omega .\end{cases}
$$

If $v$ is bounded, $v>0$ on $\bar{\Omega}$ and $u\left(x_{0}\right)>0$ for some $x_{0} \in \bar{\Omega}$, then there exists $t>0$ such that $v \equiv t u$. The same conclusion holds if $u$ is bounded, $u<0$ on $\bar{\Omega}$ and $v\left(x_{0}\right)<0$.

Proof. Suppose that $v>0$ on $\bar{\Omega}$ and $u\left(x_{0}\right)>0$. We prove the theorem trough a typical argument which is used in [6] for the linear case and Dirichlet boundary condition. Set $w_{t}=u-t v$. If $t$ is large enough $w_{t}<0$ on $\bar{\Omega}$. We define

$$
\tau=\inf \left\{t \mid w_{t}<0 \text { on } \bar{\Omega}\right\} \text {. }
$$

Clearly $w_{\tau} \leq 0$. If $\max _{\bar{\Omega}} w_{\tau}=m<0$, then for any $x \in \bar{\Omega}$

$$
w_{\tau-\epsilon}(x)=u(x)-(\tau-\epsilon) v(x) \leq m+\epsilon|v|_{\infty}<0,
$$

for $\epsilon$ small enough. This contradicts the definition of $\tau$. Then $w_{\tau}$ vanishes somewhere on $\bar{\Omega}$ and $\tau>0$ since $u\left(x_{0}\right)>0$. In conclusion $u \leq \tau v$ and $u(x)=\tau v(x)$ for some $x \in \bar{\Omega}$. Since $\tau v$ is again a supersolution, by Theorem 3.1 we have $u \equiv \tau v$.

If the inequalities satisfied by $u$ and $v$ are reversed, that is $u<0$ and $v\left(x_{0}\right)<$ 0 , we consider the function $w_{t}=t u-v$ and use the same argument.

Theorem 4.5 (Maximum principle for $\lambda<\bar{\lambda}$ ). Assume that (F1)-(F3), (f1) and (f2) hold and $\lambda<\bar{\lambda}$. Let $u \in U S C(\bar{\Omega})$ be a viscosity subsolution of

$$
\begin{cases}F\left(x, u, D u, D^{2} u\right)=\lambda u & \text { in } \Omega \\ B(x, u, D u)=0 & \text { on } \partial \Omega,\end{cases}
$$

then $u \leq 0$ on $\bar{\Omega}$.

Proof. Let $\tau \in] \lambda, \bar{\lambda}[$, then by definition there exists $v>0$ on $\bar{\Omega}$ bounded viscosity supersolution of

Then $v$ satisfies

$$
\begin{cases}F\left(x, v, D v, D^{2} v\right)=\tau v & \text { in } \quad \Omega \\ B(x, v, D v)=0 & \text { on } \quad \partial \Omega .\end{cases}
$$

$$
\begin{cases}F\left(x, v, D v, D^{2} v\right)-\lambda v \geq(\tau-\lambda) v>0 & \text { in } \quad \Omega \\ B(x, v, D v) \geq 0 & \text { on } \partial \Omega,\end{cases}
$$

in the viscosity sense. Suppose by contradiction that $u\left(x_{0}\right)>0$ for some $x_{0} \in \bar{\Omega}$. Applying Theorem 4.4 to the operator $F-\lambda I$, there exists $t>0$ such that $u \equiv t v$. Then $u$ is positive on $\bar{\Omega}$ and by homogeneity satisfies (4.4) in the viscosity sense. Since in addition $u$ is a viscosity subsolution of (4.3), using Lemma 7.3 of [19] we get

which is a contradiction.

$$
(\tau-\lambda) u \leq 0 \quad \text { in } \quad \Omega,
$$


Remark 4.6. Similarly, we can prove the minimum principle for $\lambda<\underline{\lambda}$, i.e., if $u \in \operatorname{LSC}(\bar{\Omega})$ is a viscosity supersolution of (4.3) and $\lambda<\underline{\lambda}$ then $u \geq 0$ on $\bar{\Omega}$.

Corollary 4.7. Under the assumptions of Theorem 4.5, the quantities $\bar{\lambda}$ and $\underline{\lambda}$ are finite.

Proof. By Theorem 4.5 it suffices to find $\lambda \in \mathbb{R}$ and a function $w$ which is a positive subsolution of

For

$$
\left\{\begin{array}{lll}
F\left(x, w, D w, D^{2} w\right)=\lambda w & \text { in } & \Omega \\
B(x, w, D w)=0 & \text { on } & \partial \Omega
\end{array} .\right.
$$

$$
\lambda \geq-\mathcal{M}_{a, A}^{-}\left(|f(\cdot, 1)|_{\infty}^{2} D d(x) \otimes D d(x)+|f(\cdot, 1)|_{\infty} D^{2} d(x)\right)+b|f(\cdot, 1)|_{\infty}+c,
$$

a subsolution is $w(x)=e^{|f(\cdot, 1)|_{\infty} d(x)}$.

\subsection{An example}

We want to show there exist some operators which are not proper but whose first eigenvalue $\bar{\lambda}$ is positive.

For simplicity, let us suppose that $F$ is independent of the gradient variable and that $\Omega$ is the ball of center 0 and radius R. We assume in addition that for all $(x, X) \in \bar{\Omega} \times S(N)$ and any $r>0$

$$
F(x, r, X) \geq-\mathcal{M}_{a, A}^{+}(X)+c_{0}(x) r,
$$

for some functions $c_{0}(x)$. The Isaacs operator (1.2) satisfies (4.5) if

$$
c_{\alpha, \beta}(x) \geq c_{0}(x) \text { for all } x \in \bar{\Omega} \text { and }(\alpha, \beta) \in \mathcal{A} \times \mathcal{B} .
$$

In this case the operator is proper if $c_{0}(x) \geq 0$. Since we are interested in nonproper $F$, we are looking for functions $c_{0}(x)$ in (4.5) that may be negative somewhere. We suppose that

$$
\left\{\begin{array}{lll}
c_{0}(x)>0 & \text { if } & R-\epsilon<|x| \leq R \\
c_{0}(x) \geq \beta_{1} & \text { if } & \rho<|x| \leq R-\epsilon \\
c_{0}(x) \geq-\beta_{2} & \text { if } & |x| \leq \rho,
\end{array}\right.
$$

where $0<\rho<R, \epsilon>0$ is small enough and $\beta_{1}, \beta_{2}>0$. Remark that in the ball of radius $\rho, c_{0}(x)$ may assume negative values. To prove that $\bar{\lambda}>0$ it suffices to find $v>0$ bounded supersolution of

$$
\begin{cases}-\mathcal{M}_{a, A}^{+}\left(D^{2} v\right)+c_{0}(x) v=\lambda v & \text { in } \Omega \\ B(x, v, D v)=0 & \text { on } \partial \Omega,\end{cases}
$$

for some $\lambda>0$. Assume $f(x, r) \geq 0$ for any $x \in \partial \Omega$ and $r \geq 0$, then, as shown in [23], such supersolution $v$ exists if $\beta_{1}$ and $\beta_{2}$ satisfy the following inequality for some $k>0$

$$
\beta_{2}<\frac{k e^{-k \rho} a\left(k+\frac{N-1}{\rho}\right)}{k \frac{R-\rho}{4}+k \frac{2 N A R-(N-1) a(R+\rho)}{\beta_{1} R(R-\rho)}+1-e^{-k \rho}} .
$$


As observed in [23], from the last relation we can see that choosing $k=\frac{1}{\rho}$ the term on the right-hand side goes to $+\infty$ as $\rho \rightarrow 0^{+}$, that is, if the set where $c_{0}(x)$ is negative becomes smaller then the values of $c_{0}(x)$ in this set can be very negative. On the contrary, for any value of $k$, if $\rho \rightarrow R^{-}$then $\beta_{2}$ goes to 0 . Finally, for any $k$, if $\beta_{1} \rightarrow 0^{+}$then again $\beta_{2}$ goes to 0 . So there is a sort of balance between $\beta_{1}$ and $\beta_{2}$. In [23] we present an example to explain this behavior. For operators which satisfy (4.5), the property $\bar{\lambda}>0$ can be proved in any $C^{2}$ domain, under similar assumptions on $c_{0}(x)$, see [23].

\section{Lipschitz regularity}

In this section we shall prove that viscosity solutions are Lipschitz continuous on $\bar{\Omega}$. We want to mention the works of Barles and Da Lio [5] and Milakis and Silvestre [22] about Hölder estimates of viscosity solutions of fully nonlinear elliptic equations associated to Neumann type boundary conditions.

Theorem 5.1. Assume that (F1), (F2), (F4), (f1) and (f2) hold. Let g be a bounded function and $u \in C(\bar{\Omega})$ be a viscosity solution of

$$
\begin{cases}F\left(x, u, D u, D^{2} u\right)=g(x) & \text { in } \Omega \\ B(x, u, D u)=0 & \text { on } \quad \partial \Omega,\end{cases}
$$

then there exists $C_{0}>0$ such that

$$
|u(x)-u(y)| \leq C_{0}|x-y| \quad \forall x, y \in \bar{\Omega},
$$

where $C_{0}$ depends on $N, a, A, b, c, C_{1}, \Omega$ and $|f(\cdot, u(\cdot))|_{\infty}$.

Proof. We follow the proof of Proposition III.1 of [18], that we modify taking test functions which depend on the distance function and that are suitable for the Neumann boundary conditions.

We set

$$
\Phi(x)=M K|x|-M(K|x|)^{2}
$$

and

$$
\varphi(x, y)=e^{-L(d(x)+d(y))} \Phi(x-y),
$$

where $L$ is a fixed number greater than $\frac{2}{3 r}$ with $r$ the radius in the condition $(\Omega 2)$ and $K$ and $M$ are two positive constants to be chosen later. If $K|x| \leq \frac{1}{4}$, then

$$
\Phi(x) \geq \frac{3}{4} M K|x|
$$

We define

$$
\Delta_{K}:=\left\{(x, y) \in \mathbb{R}^{N} \times \mathbb{R}^{N}:|x-y| \leq \frac{1}{4 K}\right\} .
$$

We fix $M$ such that

$$
\max _{\bar{\Omega}^{2}}|u(x)-u(y)| \leq e^{-2 L d_{0}} \frac{M}{8},
$$


where $d_{0}=\max _{x \in \bar{\Omega}} d(x)$, and we claim that taking $\delta$ small enough and $K$ large enough, one has

$$
\delta(u(x)-u(y))-\varphi(x, y) \leq 0 \quad \text { for } \quad(x, y) \in \Delta_{K} \cap \bar{\Omega}^{2} .
$$

In this case (5.1) is proven. To show (5.4) we suppose by contradiction that for some $(\bar{x}, \bar{y}) \in \Delta_{K} \cap \bar{\Omega}^{2}$

$$
\delta u(\bar{x})-\delta u(\bar{y})-\varphi(\bar{x}, \bar{y})=\max _{\Delta_{K} \cap \bar{\Omega}^{2}}(\delta u(x)-\delta u(y)-\varphi(x, y))>0 .
$$

Observe that $\delta u$ is again a solution since both $F$ and $B$ are positively homogeneous. Here we have dropped the dependence of $\bar{x}, \bar{y}$ on $K$ and $\delta$ for simplicity of notations.

Clearly $\bar{x} \neq \bar{y}$. Moreover the point $(\bar{x}, \bar{y})$ belongs to $\operatorname{int}\left(\Delta_{K}\right) \cap \bar{\Omega}^{2}$. Indeed, if $|x-y|=\frac{1}{4 K}$, by (5.3) and (5.2) for $\delta \leq 1$ we have

$$
\delta u(x)-\delta u(y) \leq|u(x)-u(y)| \leq e^{-2 L d_{0}} \frac{M}{8} \leq e^{-L(d(x)+d(y))} \frac{1}{2} M K|x-y| \leq \varphi(x, y) .
$$

Since $\bar{x} \neq \bar{y}$ we can compute the derivatives of $\varphi$ in $(\bar{x}, \bar{y})$ obtaining

$$
\begin{aligned}
D_{x} \varphi(\bar{x}, \bar{y})= & -L e^{-L(d(\bar{x})+d(\bar{y}))} M K|\bar{x}-\bar{y}|(1-K|\bar{x}-\bar{y}|) D d(\bar{x}) \\
& +e^{-L(d(\bar{x})+d(\bar{y}))} M K(1-2 K|\bar{x}-\bar{y}|) \frac{(\bar{x}-\bar{y})}{|\bar{x}-\bar{y}|}, \\
D_{y} \varphi(\bar{x}, \bar{y})= & -L e^{-L(d(\bar{x})+d(\bar{y}))} M K|\bar{x}-\bar{y}|(1-K|\bar{x}-\bar{y}|) D d(\bar{y}) \\
& -e^{-L(d(\bar{x})+d(\bar{y}))} M K(1-2 K|\bar{x}-\bar{y}|) \frac{(\bar{x}-\bar{y})}{|\bar{x}-\bar{y}|} .
\end{aligned}
$$

Observe that for $K \geq \frac{L}{4}$

$$
\left|D_{x} \varphi(\bar{x}, \bar{y})\right|,\left|D_{y} \varphi(\bar{x}, \bar{y})\right| \leq 2 M K .
$$

Using (2.1), if $\bar{x} \in \partial \Omega$ we have

$$
\begin{aligned}
B\left(\bar{x}, \delta u(\bar{x}), D_{x} \varphi(\bar{x}, \bar{y})\right)= & f(\bar{x}, \delta u(\bar{x}))+L e^{-L d(\bar{y})} M K|\bar{x}-\bar{y}|(1-K|\bar{x}-\bar{y}|) \\
& +e^{-L d(\bar{y})} M K(1-2 K|\bar{x}-\bar{y}|)\left\langle\vec{n}(\bar{x}), \frac{(\bar{x}-\bar{y})}{|\bar{x}-\bar{y}|}\right\rangle \quad(5.7) \\
\geq & \frac{1}{2} e^{-L d(\bar{y})} M K|\bar{x}-\bar{y}|\left(\frac{3}{2} L-\frac{1}{r}\right)-\delta|f(\cdot, u(\cdot))|_{\infty}>0,
\end{aligned}
$$

since $\bar{x} \neq \bar{y}, L>\frac{2}{3 r}$, for $\delta$ small enough. Similarly, if $\bar{y} \in \partial \Omega$ then $B\left(\bar{y}, \delta u(\bar{y}),-D_{y} \varphi(\bar{x}, \bar{y})\right) \leq \frac{1}{2} e^{-L d(\bar{x})} M K|\bar{x}-\bar{y}|\left(-\frac{3}{2} L+\frac{1}{r}\right)+\delta|f(\cdot, u(\cdot))|_{\infty}<0$.

Then $\bar{x}, \bar{y} \in \Omega$ and

$$
\begin{gathered}
F\left(\bar{x}, \delta u(\bar{x}), D_{x} \varphi(\bar{x}, \bar{y}), X\right) \leq \delta g(\bar{x}), \quad \text { if } \quad\left(D_{x} \varphi(\bar{x}, \bar{y}), X\right) \in \bar{J}^{2,+} \delta u(\bar{x}), \\
F\left(\bar{y}, \delta u(\bar{y}),-D_{y} \varphi(\bar{x}, \bar{y}), Y\right) \geq \delta g(\bar{y}) \quad \text { if } \quad\left(-D_{y} \varphi(\bar{x}, \bar{y}), Y\right) \in \bar{J}^{2,-} \delta u(\bar{y}) .
\end{gathered}
$$


Since $(\bar{x}, \bar{y}) \in \operatorname{int} \Delta_{K} \cap \bar{\Omega}^{2}$, it is a local maximum point of $\delta u(x)-\delta u(y)-\varphi(x, y)$ in $\bar{\Omega}^{2}$. Then applying Theorem 3.2 in [11], for every $\epsilon>0$ there exist $X, Y \in S(N)$ such that $\left(D_{x} \varphi(\bar{x}, \bar{y}), X\right) \in \bar{J}^{2,+} \delta u(\bar{x}),\left(-D_{y} \varphi(\bar{x}, \bar{y}), Y\right) \in \bar{J}^{2,-} \delta u(\bar{y})$ and

$$
\left(\begin{array}{cc}
X & 0 \\
0 & -Y
\end{array}\right) \leq D^{2}(\varphi(\bar{x}, \bar{y}))+\epsilon\left(D^{2}(\varphi(\bar{x}, \bar{y}))\right)^{2} .
$$

Now we want to estimate the matrix on the right-hand side of the last inequality.

$$
\begin{aligned}
D^{2} \varphi(\bar{x}, \bar{y})= & \Phi(\bar{x}-\bar{y}) D^{2}\left(e^{-L(d(\bar{x})+d(\bar{y}))}\right)+D\left(e^{-L(d(\bar{x})+d(\bar{y}))}\right) \otimes D(\Phi(\bar{x}-\bar{y})) \\
& +D(\Phi(\bar{x}-\bar{y})) \otimes D\left(e^{-L(d(\bar{x})+d(\bar{y}))}\right)+e^{-L(d(\bar{x})+d(\bar{y}))} D^{2}(\Phi(\bar{x}-\bar{y})) .
\end{aligned}
$$

We set

$$
\begin{aligned}
& A_{1}:=\Phi(\bar{x}-\bar{y}) D^{2}\left(e^{-L(d(\bar{x})+d(\bar{y}))}\right), \\
& A_{2}:=D\left(e^{-L(d(\bar{x})+d(\bar{y}))}\right) \otimes D(\Phi(\bar{x}-\bar{y}))+D(\Phi(\bar{x}-\bar{y})) \otimes D\left(e^{-L(d(\bar{x})+d(\bar{y}))}\right), \\
& A_{3}:=e^{-L(d(\bar{x})+d(\bar{y}))} D^{2}(\Phi(\bar{x}-\bar{y})) .
\end{aligned}
$$

Observe that

$$
A_{1} \leq C K|\bar{x}-\bar{y}|\left(\begin{array}{cc}
I & 0 \\
0 & I
\end{array}\right) .
$$

Here and henceforth $\mathrm{C}$ denotes various positive constants independent of $K$ and $\delta$.

For $A_{2}$ we have the following estimate

$$
A_{2} \leq C K\left(\begin{array}{cc}
I & 0 \\
0 & I
\end{array}\right)+C K\left(\begin{array}{cc}
I & -I \\
-I & I
\end{array}\right) .
$$

Indeed for $\xi, \eta \in \mathbb{R}^{N}$ we compute

$$
\begin{aligned}
\left\langle A_{2}(\xi, \eta),(\xi, \eta)\right\rangle= & 2 L e^{-L(d(\bar{x})+d(\bar{y}))}\{\langle D d(\bar{x}) \otimes D \Phi(\bar{x}-\bar{y})(\eta-\xi), \xi\rangle \\
& +\langle D d(\bar{y}) \otimes D \Phi(\bar{x}-\bar{y})(\eta-\xi), \eta\rangle\} \leq C K(|\xi|+|\eta|)|\eta-\xi| \\
\leq & C K\left(|\xi|^{2}+|\eta|^{2}\right)+C K|\eta-\xi|^{2} .
\end{aligned}
$$

Now we consider $A_{3}$. The matrix $D^{2}(\Phi(\bar{x}-\bar{y}))$ has the form

$$
D^{2}(\Phi(\bar{x}-\bar{y}))=\left(\begin{array}{cc}
D^{2} \Phi(\bar{x}-\bar{y}) & -D^{2} \Phi(\bar{x}-\bar{y}) \\
-D^{2} \Phi(\bar{x}-\bar{y}) & D^{2} \Phi(\bar{x}-\bar{y})
\end{array}\right),
$$

and the Hessian matrix of $\Phi(x)$ is

$$
D^{2} \Phi(x)=\frac{M K}{|x|}\left(I-\frac{x \otimes x}{|x|^{2}}\right)-2 M K^{2} I .
$$

If we choose

$$
\epsilon=\frac{|\bar{x}-\bar{y}|}{2 M K e^{-L(d(\bar{x})+d(\bar{y}))}},
$$


then we have the following estimates

$$
\begin{gathered}
\epsilon A_{1}^{2} \leq C K|\bar{x}-\bar{y}|^{3} I_{2 N}, \quad \epsilon A_{2}^{2} \leq C K|\bar{x}-\bar{y}| I_{2 N}, \\
\epsilon\left(A_{1} A_{2}+A_{2} A_{1}\right) \leq C K|\bar{x}-\bar{y}|^{2} I_{2 N}, \\
\epsilon\left(A_{1} A_{3}+A_{3} A_{1}\right) \leq C K|\bar{x}-\bar{y}| I_{2 N}, \quad \epsilon\left(A_{2} A_{3}+A_{3} A_{2}\right) \leq C K I_{2 N},
\end{gathered}
$$

where $I_{2 N}:=\left(\begin{array}{ll}I & 0 \\ 0 & I\end{array}\right)$. Then using (5.9), (5.10), (5.13) and observing that

$$
\left(D^{2}(\Phi(\bar{x}-\bar{y}))\right)^{2}=\left(\begin{array}{cc}
2\left(D^{2} \Phi(\bar{x}-\bar{y})\right)^{2} & -2\left(D^{2} \Phi(\bar{x}-\bar{y})\right)^{2} \\
-2\left(D^{2} \Phi(\bar{x}-\bar{y})\right)^{2} & 2\left(D^{2} \Phi(\bar{x}-\bar{y})\right)^{2}
\end{array}\right),
$$

from (5.8) we can conclude that

$$
\left(\begin{array}{cc}
X & 0 \\
0 & -Y
\end{array}\right) \leq O(K)\left(\begin{array}{ll}
I & 0 \\
0 & I
\end{array}\right)+\left(\begin{array}{cc}
B & -B \\
-B & B
\end{array}\right)
$$

where

$$
B=C K I+e^{-L(d(\bar{x})+d(\bar{y}))}\left[D^{2} \Phi(\bar{x}-\bar{y})+\frac{|\bar{x}-\bar{y}|}{M K}\left(D^{2} \Phi(\bar{x}-\bar{y})\right)^{2}\right] .
$$

The last inequality can be rewritten as follows

$$
\left(\begin{array}{cc}
\widetilde{X} & 0 \\
0 & -\widetilde{Y}
\end{array}\right) \leq\left(\begin{array}{cc}
B & -B \\
-B & B
\end{array}\right)
$$

with $\widetilde{X}=X-O(K) I$ and $\tilde{Y}=Y+O(K) I$.

Now we want to get a good estimate for $\operatorname{tr}(\tilde{X}-\tilde{Y})$, as in [18]. For that aim let

$$
0 \leq P:=\frac{(\bar{x}-\bar{y}) \otimes(\bar{x}-\bar{y})}{|\bar{x}-\bar{y}|^{2}} \leq I .
$$

Since $\widetilde{X}-\tilde{Y} \leq 0$ and $\widetilde{X}-\tilde{Y} \leq 4 B$, we have

$$
\operatorname{tr}(\tilde{X}-\tilde{Y}) \leq \operatorname{tr}(P(\widetilde{X}-\tilde{Y})) \leq 4 \operatorname{tr}(P B) .
$$

We have to compute $\operatorname{tr}(P B)$. From (5.11), observing that the matrix $\left(1 /|x|^{2}\right) x \otimes x$ is idempotent, i.e., $\left[\left(1 /|x|^{2}\right) x \otimes x\right]^{2}=\left(1 /|x|^{2}\right) x \otimes x$, we compute

$$
\left(D^{2} \Phi(x)\right)^{2}=\frac{M^{2} K^{2}}{|x|^{2}}(1-4 K|x|)\left(I-\frac{x \otimes x}{|x|^{2}}\right)+4 M^{2} K^{4} I .
$$

Then, since $\operatorname{tr} P=1$ and $4 K|\bar{x}-\bar{y}| \leq 1$, we have

$$
\begin{aligned}
\operatorname{tr}(P B) & =C K+e^{-L(d(\bar{x})+d(\bar{y}))}\left(-2 M K^{2}+4 M K^{3}|\bar{x}-\bar{y}|\right) \\
& \leq C K-e^{-L(d(\bar{x})+d(\bar{y}))} M K^{2}<0,
\end{aligned}
$$

for large $K$. This gives

$$
|\operatorname{tr}(\tilde{X}-\tilde{Y})|=-\operatorname{tr}(\tilde{X}-\tilde{Y}) \geq 4 e^{-L(d(\bar{x})+d(\bar{y}))} M K^{2}-4 C K \geq C K^{2},
$$


for large $K$. Since $\|B\| \leq \frac{C K}{|\bar{x}-\bar{y}|}$, we have

$$
\|B\|^{\frac{1}{2}}|\operatorname{tr}(\tilde{X}-\tilde{Y})|^{\frac{1}{2}} \leq\left(\frac{C K}{|\bar{x}-\bar{y}|}\right)^{\frac{1}{2}}|\operatorname{tr}(\tilde{X}-\tilde{Y})|^{\frac{1}{2}} \leq \frac{C}{K^{\frac{1}{2}}|\bar{x}-\bar{y}|^{\frac{1}{2}}}|\operatorname{tr}(\tilde{X}-\tilde{Y})| .
$$

The Lemma III.I in [18] ensures the existence of a universal constant $C$ depending only on $N$ such that

$$
\|\widetilde{X}\|,\|\widetilde{Y}\| \leq C\left\{|\operatorname{tr}(\widetilde{X}-\tilde{Y})|+\|B\|^{\frac{1}{2}}|\operatorname{tr}(\tilde{X}-\tilde{Y})|^{\frac{1}{2}}\right\} .
$$

Thanks to the above estimates we can conclude that

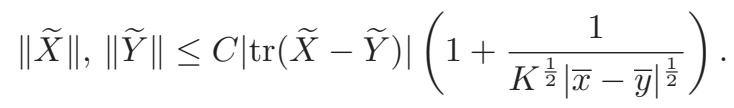

Now, using assumptions (F2) and (F4) concerning $F$, the definition of $\widetilde{X}$ and $\tilde{Y}$ and the fact that $\delta u$ is sub and supersolution we compute

$$
\begin{aligned}
\delta g(\bar{y}) \leq & F\left(\bar{y}, \delta u(\bar{y}),-D_{y} \varphi, Y\right) \leq F\left(\bar{y}, \delta u(\bar{y}),-D_{y} \varphi, \widetilde{Y}\right)+O(K) \\
\leq & F\left(\bar{y}, \delta u(\bar{x}), D_{x} \varphi, \widetilde{X}\right)+c \delta|u(\bar{x})-u(\bar{y})|+b\left|D_{x} \varphi+D_{y} \varphi\right| \\
& +a \operatorname{tr}(\widetilde{X}-\widetilde{Y})+O(K) \\
\leq & F\left(\bar{x}, \delta u(\bar{x}), D_{x} \varphi, \widetilde{X}\right)+2 c \delta|u(\bar{x})|+2 b\left|D_{x} \varphi\right|+C_{1}|\bar{x}-\bar{y}|^{\frac{1}{2}}\|\widetilde{X}\| \\
& +c \delta|u(\bar{x})-u(\bar{y})|+b\left|D_{x} \varphi+D_{y} \varphi\right|+a \operatorname{tr}(\tilde{X}-\tilde{Y})+O(K) \\
\leq & \delta g(\bar{x})+2 c \delta|u(\bar{x})|+2 b\left|D_{x} \varphi\right|+C_{1}|\bar{x}-\bar{y}|^{\frac{1}{2}}\|\widetilde{X}\|+c \delta|u(\bar{x})-u(\bar{y})| \\
& +b\left|D_{x} \varphi+D_{y} \varphi\right|+a \operatorname{tr}(\widetilde{X}-\tilde{Y})+O(K) .
\end{aligned}
$$

From this inequalities, using (5.6) and (5.15), we get

$$
\begin{aligned}
& \delta g(\bar{y})- \delta g(\bar{x})-2 c \delta|u(\bar{x})|-c \delta|u(\bar{x})-u(\bar{y})| \\
& \leq O(K)+C|\operatorname{tr}(\tilde{X}-\tilde{Y})|\left(|\bar{x}-\bar{y}|^{\frac{1}{2}}+K^{-\frac{1}{2}}\right)+a \operatorname{tr}(\tilde{X}-\tilde{Y}) \\
& \quad=\operatorname{atr}(\tilde{X}-\tilde{Y})+o(|\operatorname{tr}(\tilde{X}-\tilde{Y})|),
\end{aligned}
$$

as $K \rightarrow+\infty$. Since $g$ and $u$ are bounded, the first member in (5.16) is bounded from below by the quantity $-2|g|_{\infty}-4 c|u|_{\infty}$ which is independent of $\delta$. But the last term in (5.16) goes to $-\infty$ as $K \rightarrow+\infty$, hence taking $K$ so large that

$$
a \operatorname{tr}(\tilde{X}-\tilde{Y})+o(|\operatorname{tr}(\widetilde{X}-\tilde{Y})|)<-2|g|_{\infty}-4 c|u|_{\infty},
$$

and then $\delta$ so small that the last member in (5.7) is positive, we obtain a contradiction and this concludes the proof.

Remark 5.2. The regularity theorem can be shown also for solutions of the Neumann problem for the operator

$$
\sup _{\alpha \in \mathcal{A}} \inf _{\beta \in \mathcal{B}}\left\{-\operatorname{tr}\left(A_{\alpha, \beta}(x) D^{2} u\right)+b_{\alpha, \beta}(x) \cdot D u+c_{\alpha, \beta}(x) u-g_{\alpha, \beta}(x)\right\},
$$

if the functions $g_{\alpha, \beta}$ are bounded uniformly in $\alpha$ and $\beta$. 
Since the Lipschitz estimate depends only on the bounds of the solution of $g$ and on the structural constants, an immediate consequence of the previous theorem is the following compactness criterion that will be useful in the next sections.

Corollary 5.3. Assume the same hypothesis of Theorem 5.1. Suppose that $\left(g_{n}\right)_{n}$ is a sequence of continuous and uniformly bounded functions and $\left(u_{n}\right)_{n}$ is a sequence of uniformly bounded viscosity solutions of

$$
\begin{cases}F\left(x, u_{n}, D u_{n}, D^{2} u_{n}\right)=g_{n}(x) & \text { in } \Omega \\ B\left(x, u_{n}, D u_{n}\right)=0 & \text { on } \partial \Omega .\end{cases}
$$

Then the sequence $\left(u_{n}\right)_{n}$ is relatively compact in $C(\bar{\Omega})$.

\section{Existence results}

This section is devoted to the problem of the existence of a solution of

$$
\begin{cases}F\left(x, u, D u, D^{2} u\right)=\lambda u+g(x) & \text { in } \Omega \\ B(x, u, D u)=0 & \text { on } \partial \Omega .\end{cases}
$$

Using the well known result which guarantees that (6.1) with $\lambda=0$ is uniquely solvable if $F$ satisfies

(F5) There exists $\sigma>0$ such that for any $(x, p, X) \in \bar{\Omega} \times \mathbb{R}^{N} \times S(N)$ the function $r \rightarrow F(x, r, p, X)-\sigma r$ is non-decreasing on $\mathbb{R}$,

see [11] Theorem 7.5, we will prove the existence of a positive solution of (6.1) when $g$ is non-negative and $\lambda<\bar{\lambda}$, without requiring (F5). The solution is unique if $g>0$. Then we will show the existence of a positive principal eigenfunction corresponding to $\bar{\lambda}$, that is a solution of (6.1) when $g \equiv 0$ and $\lambda=\bar{\lambda}$. For the last two results we will follow the proof given in [8] for the analogous theorems with the Dirichlet boundary condition.

Symmetrical results can be obtained for the eigenvalue $\underline{\lambda}$.

Finally, we will prove that the Neumann problem (6.1) is solvable for any right-hand side if $\lambda<\min \{\bar{\lambda}, \underline{\lambda}\}$.

The following is a well known result, see [11] Theorem 7.5.

Theorem 6.1. Suppose that (F2), (F3), (F5), (f1) and (f3) hold and that $g$ is continuous on $\bar{\Omega}$. If $u \in U S C(\bar{\Omega})$ and $v \in L S C(\bar{\Omega})$ are respectively sub and supersolution of

$$
\begin{cases}F\left(x, u, D u, D^{2} u\right)=g(x) & \text { in } \Omega \\ B(x, u, D u)=0 & \text { on } \quad \partial \Omega,\end{cases}
$$

then $u \leq v$ on $\bar{\Omega}$. Moreover (6.2) has a unique viscosity solution. 
Theorem 6.2. Assume that (F1)-(F3), (f1) and (f2) hold. Suppose $h \geq 0, g \leq h$ and $g(x)<0$ if $h(x)=0$. Let $u \in U S C(\bar{\Omega})$ be a viscosity subsolution of

$$
\begin{cases}F\left(x, u, D u, D^{2} u\right)=\lambda u+g(x) & \text { in } \Omega \\ B(x, u, D u)=0 & \text { on } \quad \partial \Omega\end{cases}
$$

and let $v \in L S C(\bar{\Omega})$ be a bounded positive viscosity supersolution of

$$
\begin{cases}F\left(x, v, D v, D^{2} v\right)=\lambda v+h(x) & \text { in } \Omega \\ B(x, v, D v)=0 & \text { on } \quad \partial \Omega .\end{cases}
$$

Then $u \leq v$ on $\bar{\Omega}$.

Remark 6.3. The existence of such a $v$ implies $\lambda \leq \bar{\lambda}$.

Remark 6.4. Similarly, we can prove the comparison result between $u$ and $v$ if $u$ is negative and bounded, $g \leq 0, g \leq h$ and $h(x)>0$ if $g(x)=0$.

Proof. Suppose by contradiction that $\max _{\bar{\Omega}}(u-v)=u(\bar{x})-v(\bar{x})>0$ for some $\bar{x} \in \bar{\Omega}$. Set $w_{t}=u-t v$. If $t$ is large enough $w_{t}<0$ on $\bar{\Omega}$. We define

$$
\tau=\inf \left\{t \mid w_{t}<0 \text { on } \bar{\Omega}\right\} .
$$

As in the proof of Theorem 4.4, $w_{\tau} \leq 0$ and vanishes in some point, i.e., $u \leq \tau v$ and $u(x)=\tau v(x)$ for some $x \in \bar{\Omega}$. Moreover, since $u(\bar{x})>v(\bar{x})$ we know that $\tau>1$, which implies that $h \leq \tau h$, being $h$ non-negative. Then $\tau v$ is still a supersolution of (6.3) and $u \equiv \tau v$ by Theorem 3.1. Hence, applying Lemma 7.3 of [19] we get

$$
\tau h \leq g,
$$

which contradicts the assumptions on $g$ and $h$.

Theorem 6.5. Suppose that (F1)-(F4), (f1)-(f3) hold, that $\lambda<\bar{\lambda}, g \geq 0, g \neq \equiv$ and $g$ is continuous on $\bar{\Omega}$, then there exists a positive viscosity solution of (6.1). The positive solution is unique if $g>0$.

Proof. The condition (F2) implies that $r \rightarrow F(x, r, p, X)+c r$ is non-decreasing. Hence the operator $F+(2 c+|\lambda|) I$ satisfies (F5) with $\sigma=c+|\lambda|$, so that by Theorem 6.1 the sequence $\left(u_{n}\right)_{n}$ defined by $u_{1}=0$ and $u_{n+1}$ as the solution of

$$
\begin{cases}F\left(x, u_{n+1}, D u_{n+1}, D^{2} u_{n+1}\right)+(2 c+|\lambda|) u_{n+1}=g+(2 c+|\lambda|+\lambda) u_{n} & \text { in } \Omega \\ B\left(x, u_{n+1}, D u_{n+1}\right)=0 & \text { on } \partial \Omega,\end{cases}
$$

is well defined. By the comparison Theorems 6.1 and 3.1 , since $g \geq 0$ and $g \not \equiv 0$ the sequence is positive and increasing.

We use the argument of Theorem 7 of [8] to prove that $\left(u_{n}\right)_{n}$ is also bounded. Suppose that it is not, then dividing by $\left|u_{n+1}\right|_{\infty}$ and defining $v_{n}:=\frac{u_{n}}{\left|u_{n}\right|_{\infty}}$ one gets 
that $v_{n+1}$ is a solution of

$$
\begin{cases}F\left(x, v_{n+1}, D v_{n+1}, D^{2} v_{n+1}\right)+(2 c+|\lambda|) v_{n+1} & \\ \quad=\frac{g}{\left|u_{n+1}\right|_{\infty}}+(2 c+|\lambda|+\lambda) \frac{u_{n}}{\left|u_{n+1}\right|_{\infty}} & \text { in } \Omega \\ B\left(x, v_{n+1}, D v_{n+1}\right)=0 & \text { on } \quad \partial \Omega .\end{cases}
$$

By Corollary 5.3, $\left(v_{n}\right)_{n}$ converges along a subsequence to a positive function $v$ which satisfies

$$
\begin{cases}F\left(x, v, D v, D^{2} v\right)-\lambda v=(2 c+|\lambda|+\lambda)(k-1) v \leq 0 & \text { in } \Omega \\ B(x, v, D v)=0 & \text { on } \partial \Omega,\end{cases}
$$

where $k:=\lim \sup _{n \rightarrow+\infty} \frac{\left|u_{n}\right|_{\infty}}{\left|u_{n+1}\right|_{\infty}} \leq 1$. This contradicts the maximum principle, Theorem 4.5. Then $\left(u_{n}\right)_{n}$ is bounded and letting $n$ go to infinity, by the compactness result, the sequence converges uniformly to a function $u$ which is a solution. Moreover the solution is positive on $\bar{\Omega}$ by Corollary 3.4 .

The uniqueness of the positive solution follows from Theorem 6.2.

Theorem 6.6 (Existence of principal eigenfunctions). Suppose that (F1)-(F4), (f1)-(f3) hold. Then there exists $\phi>0$ on $\bar{\Omega}$ viscosity solution of

$$
\begin{cases}F\left(x, \phi, D \phi, D^{2} \phi\right)=\bar{\lambda} \phi & \text { in } \quad \Omega \\ B(x, \phi, D \phi)=0 & \text { on } \quad \partial \Omega .\end{cases}
$$

Moreover $\phi$ is Lipschitz continuous on $\bar{\Omega}$.

Proof. Let $\lambda_{n}$ be an increasing sequence which converges to $\bar{\lambda}$. Let $u_{n}$ be a positive solution of

$$
\begin{cases}F\left(x, u_{n}, D u_{n}, D^{2} u_{n}\right)=\lambda_{n} u_{n}+1 & \text { in } \Omega \\ B\left(x, u_{n}, D u_{n}\right)=0 & \text { on } \partial \Omega .\end{cases}
$$

By Theorem 6.5 the sequence $\left(u_{n}\right)_{n}$ is well defined. Following the argument of the proof of Theorem 8 of [8] we can prove that it is unbounded, otherwise one would contradict the definition of $\bar{\lambda}$. Then, up to subsequence, $\left|u_{n}\right|_{\infty} \rightarrow+\infty$ as $n \rightarrow+\infty$ and defining $v_{n}:=\frac{u_{n}}{\left|u_{n}\right|_{\infty}}$ one gets that $v_{n}$ satisfies

$$
\begin{cases}F\left(x, v_{n}, D v_{n}, D^{2} v_{n}\right)=\lambda_{n} v_{n}+\frac{1}{\left|u_{n}\right|_{\infty}} & \text { in } \quad \Omega \\ B\left(x, v_{n}, D v_{n}\right)=0 & \text { on } \quad \partial \Omega .\end{cases}
$$

Then, by Corollary 5.3, we can extract a subsequence converging to a function $\phi$ with $|\phi|_{\infty}=1$ which is positive on $\bar{\Omega}$ by Corollary 3.4 and is the desired solution. By Theorem 5.1 the solution is also Lipschitz continuous on $\bar{\Omega}$.

Remark 6.7. With the same arguments used in the proofs of Theorems 6.5 and 6.6 one can prove: the existence of a negative viscosity solution of (6.1), for $\lambda<\underline{\lambda}$ and 
$g \leq 0, g \not \equiv 0$, which is unique if $g<0$ by Remark 6.4; the existence of a negative Lipschitz principal eigenfunction corresponding to $\underline{\lambda}$, i.e., a solution of

$$
\left\{\begin{array}{lll}
F\left(x, \phi, D \phi, D^{2} \phi\right)=\underline{\lambda} \phi & \text { in } & \Omega \\
B(x, \phi, D \phi)=0 & \text { on } & \partial \Omega
\end{array} .\right.
$$

Theorem 6.8. Suppose that (F1)-(F4), (f1)-(f3) hold. Suppose that $\lambda<\min \{\bar{\lambda}, \underline{\lambda}\}$ and $g$ is continuous on $\bar{\Omega}$, then there exists a viscosity solution of (6.1).

Proof. If $g \equiv 0$, by the maximum and minimum principles the only solution is $u \equiv 0$. Let us suppose $g \not \equiv 0$. Since $\lambda<\min \{\bar{\lambda}, \underline{\lambda}\}$ by Theorem 6.5 and Remark 6.7 there exist $v_{0} \in C(\bar{\Omega})$ positive viscosity solution of (6.1) with right-hand side $|g|_{\infty}$ and $u_{0} \in C(\bar{\Omega})$ negative viscosity solution of (6.1) with right-hand side $-|g|_{\infty}$.

Let $\left(u_{n}\right)_{n}$ be the sequence defined in the proof of Theorem 6.5 with $u_{1}=u_{0}$. By comparison Theorem 6.1 we have $u_{0}=u_{1} \leq u_{2} \leq \cdots \leq v_{0}$. Hence, by the compactness Corollary 5.3 the sequence converges to a continuous function which is the desired solution.

Remark 6.9. The existence results can be shown also for the operator

$$
\sup _{\alpha \in \mathcal{A}} \inf _{\beta \in \mathcal{B}}\left\{-\operatorname{tr}\left(A_{\alpha, \beta}(x) D^{2} u\right)+b_{\alpha, \beta}(x) \cdot D u+c_{\alpha, \beta}(x) u-g_{\alpha, \beta}(x)\right\},
$$

if the functions $g_{\alpha, \beta}$ are continuous uniformly in $\alpha$ and $\beta$. In particular, in that case, if $\bar{\lambda}$ and $\underline{\lambda}$ are positive there exists a viscosity solution of

$$
\left\{\begin{array}{lr}
\sup _{\alpha \in \mathcal{A}} \inf _{\beta \in \mathcal{B}}\left\{-\operatorname{tr}\left(A_{\alpha, \beta}(x) D^{2} u\right)+b_{\alpha, \beta}(x) \cdot D u+c_{\alpha, \beta}(x) u-g_{\alpha, \beta}(x)\right\}=0 & \text { in } \Omega \\
B(x, u, D u)=0 & \text { on } \partial \Omega .
\end{array}\right.
$$

\section{Properties of the principal eigenvalues}

In this section we establish some of the basic properties of the principal eigenvalues. We denote by $\phi^{+}$a positive eigenfunction corresponding to $\bar{\lambda}$ and by $\phi^{-}$a negative eigenfunction corresponding to $\underline{\lambda}$. Throughout this section we assume (F1)-(F4) and (f1)-(f3).

The next result states that the principal eigenfunctions are simple, in the sense that they are equal up to a multiplicative constant.

Proposition 7.1. If $u \in U S C(\bar{\Omega})$ is a viscosity subsolution of

$$
\begin{cases}F\left(x, u, D u, D^{2} u\right)=\bar{\lambda} u & \text { in } \Omega \\ B(x, u, D u)=0 & \text { on } \partial \Omega,\end{cases}
$$

and $u\left(x_{0}\right)>0$ for some $x_{0} \in \bar{\Omega}$ then there exists $t>0$ such that $u \equiv t \phi^{+}$. If $u \in L S C(\bar{\Omega})$ is a viscosity supersolution of (7.1) with $\bar{\lambda}$ replaced by $\underline{\lambda}$ and $u\left(x_{0}\right)<0$, then there exists $t>0$ such that $u \equiv t \phi^{-}$. 
Assume in addition

$$
\begin{aligned}
& -F(x,-r,-p,-X) \leq F(x, r, p, X) \\
& \qquad \text { for any }(x, r, p, X) \in \bar{\Omega} \times \mathbb{R} \times \mathbb{R}^{N} \times \mathrm{S}(\mathrm{N})
\end{aligned}
$$

and

$$
-f(x,-r) \leq f(x, r) \quad \text { for any } \quad(x, r) \in \partial \Omega \times \mathbb{R} .
$$

If $u \in C(\bar{\Omega}), u \neq \equiv$, is a viscosity subsolution of (7.1) then there exists $t \in \mathbb{R}$ such that $u \equiv t \phi^{+}$. If $u \in C(\bar{\Omega}), u \not \equiv 0$ is a viscosity solution of (7.1) with $\bar{\lambda}$ replaced by $\underline{\lambda}$, there exists $t \in \mathbb{R}$ such that $u \equiv t \phi^{-}$.

Proof. If $u$ is a subsolution (resp., supersolution) of (7.1) (resp., of (7.1) with $\underline{\lambda}$ instead of $\bar{\lambda}$ ) and $u\left(x_{0}\right)>0$ (resp., $u\left(x_{0}\right)<0$ ), then by Theorem 4.4 we have $u \equiv t \phi^{+}$(resp., $u \equiv t \phi^{-}$) for some $t>0$.

Now assume (7.2)-(7.3) and let $u \not \equiv 0$ be a subsolution of (7.1). If $u$ is positive somewhere we are in the previous case. If $u$ is negative on $\bar{\Omega}$ then the function $w:=-u$ is a positive continuous supersolution of

$$
\begin{cases}F\left(x, w, D w, D^{2} w\right)-\bar{\lambda} w \geq-F\left(x,-w,-D w,-D^{2} w\right)+\bar{\lambda}(-w) \geq 0 & \text { in } \Omega \\ B(x, w, D w) \geq-B(x,-w,-D w) \geq 0 & \text { on } \partial \Omega .\end{cases}
$$

Hence, again from Theorem 4.4 it follows that $u \equiv t \phi^{+}$, for some $t<0$.

Finally, let $u \not \equiv 0$ be a solution of (7.1) with $\underline{\lambda}$ instead of $\bar{\lambda}$. Remark that conditions (7.2)-(7.3) imply $\underline{\lambda} \leq \bar{\lambda}$. If $\underline{\lambda}<\bar{\lambda}$, then by the maximum principle, Theorem 4.5, $u<0$ on $\bar{\Omega}$ and we are in the first case. If $\underline{\lambda}=\bar{\lambda}$, by the simplicity of $\bar{\lambda}$ just proved, $u \equiv t \phi^{-}$for some $t<0$.

Remark 7.2. If $F$ and $f$ satisfy

$$
-F(x,-r,-p,-X) \geq F(x, r, p, X) \quad \text { for any } \quad(x, r, p, X) \in \bar{\Omega} \times \mathbb{R} \times \mathbb{R}^{N} \times S(N)
$$

and

$$
-f(x,-r) \geq f(x, r) \text { for any }(x, r) \in \partial \Omega \times \mathbb{R},
$$

then, applying Proposition 7.1 to the operator $G(x, r, p, X)=-F(x,-r,-p,-X)$ with $B(x, r, p)=\widetilde{f}(x, r)+\langle p, \vec{n}(x)\rangle$, where $\widetilde{f}(x, r)=-f(x,-r)$, we get again simplicity of principal eigenvalues.

Remark 7.3. Convex and 1-homogeneous operators satisfy the assumption (7.2).

Proposition 7.4. $\bar{\lambda}$ (resp., $\underline{\lambda}$ ) is the only eigenvalue corresponding to a positive (resp., negative) eigenfunction.

Proof. Let $u$ be a positive eigenfunction corresponding to $\mu$. By the definition of $\bar{\lambda}$, we have $\mu \leq \bar{\lambda}$. If $\mu<\bar{\lambda}$, we must have $u \leq 0$ by Theorem 4.5 , which is a contradiction. Thus $\mu=\lambda$.

The following proposition states that the principal eigenvalues are isolated. 
Proposition 7.5. There exists $\epsilon>0$ such that the problem

$$
\begin{cases}F\left(x, u, D u, D^{2} u\right)=\lambda u & \text { in } \Omega \\ B(x, u, D u)=0 & \text { on } \partial \Omega,\end{cases}
$$

has no solutions $u \not \equiv 0$, for $\lambda \in(-\infty, \max \{\underline{\lambda}, \bar{\lambda}\}+\epsilon) \backslash\{\bar{\lambda}, \underline{\lambda}\}$.

Proof. We may suppose without loss of generality that $\bar{\lambda} \leq \underline{\lambda}$. If $\lambda<\bar{\lambda} \leq \underline{\lambda}$ then it follows from the maximum and minimum principles that $u \equiv 0$ is the only solution of (7.4).

If $\lambda<\underline{\lambda}$ and $u \not \equiv 0$ is a solution of (7.4), by the minimum principle we have $u>0$ on $\bar{\Omega}$. Then Proposition 7.4 implies $\lambda=\bar{\lambda}$.

Finally suppose that there exists a sequence $\lambda_{n} \downarrow \underline{\lambda}$ such that the problem (7.4) with $\lambda=\lambda_{n}$ has a solution $\phi_{n} \not \equiv 0$. We can assume that $\left|\phi_{n}\right|_{\infty}=1$ for any $n$. Then by the compactness criterion, Corollary 5.3, the sequence $\left(\phi_{n}\right)_{n}$ converges uniformly on $\bar{\Omega}$ to a function $\phi \not \equiv 0$ which is a solution of (7.4) with $\lambda=\underline{\lambda}$. By Proposition 7.4 the functions $\phi_{n}$ change sign in $\Omega$ while by Proposition 7.1 and Theorem 3.1 either $\phi>0$ or $\phi<0$ on $\bar{\Omega}$. This contradicts the uniform convergence of $\left(\phi_{n}\right)_{n}$ to $\phi$.

We want to conclude this section with the following comparison, suggested by Hitoshi Ishii [17], between $\bar{\lambda}=\bar{\lambda}_{N}$ and $\bar{\lambda}_{D}$ respectively the principal eigenvalues corresponding to the Neumann and the Dirichlet problems.

Proposition 7.6. $\bar{\lambda}_{N}<\bar{\lambda}_{D}$.

Proof. Let $v$ and $w$ be respectively the eigenfunctions corresponding to $\bar{\lambda}_{N}$ and $\bar{\lambda}_{D}$. That is

$$
\begin{aligned}
& F\left(x, v, D v, D^{2} v\right)=\bar{\lambda}_{N} v \quad \text { in } \quad \Omega, \quad B(x, v, D v)=0 \quad \text { on } \quad \partial \Omega, \quad v>0 \quad \text { on } \bar{\Omega}, \\
& F\left(x, w, D w, D^{2} w\right)=\bar{\lambda}_{D} w \quad \text { in } \quad \Omega, \quad w=0 \quad \text { on } \quad \partial \Omega, \quad w>0 \quad \text { in } \quad \Omega \text {. }
\end{aligned}
$$

Since $f(x, 0)=0$, we see that $w$ satisfies

$$
F\left(x, w, D w, D^{2} w\right)=\bar{\lambda}_{D} w \quad \text { in } \quad \Omega, \quad B(x, w, D w) \leq 0 \quad \text { on } \quad \partial \Omega .
$$

Let us suppose $\bar{\lambda}_{N} \geq \bar{\lambda}_{D}$. Then

$$
F\left(x, w, D w, D^{2} w\right) \leq \bar{\lambda}_{N} w \quad \text { in } \quad \Omega, \quad B(x, w, D w) \leq 0 \quad \text { on } \quad \partial \Omega .
$$

Replacing $w$ by its constant multiple $t w$ with $t>0$, we may assume that $w \leq v$ on $\bar{\Omega}$ and $w\left(x_{0}\right)=v\left(x_{0}\right)$ for some $x_{0} \in \Omega$. Note that $w(x)=0<v(x)$ for all $x \in \partial \Omega$. By Theorem 3.2 we must have $w \equiv v$ or $w<v$ on $\bar{\Omega}$. This is a contradiction.

\section{The Pucci's operators}

In this section we want to show that the two principal eigenvalues of the following operator

$$
F\left(x, u, D u, D^{2} u\right)=-\mathcal{M}_{a, A}^{+}\left(D^{2} u\right)+b(x) \cdot D u+c(x) u,
$$


with the pure Neumann boundary condition may be different. Suppose $b \in C^{0,1}(\bar{\Omega})$, $c \in C^{0, \beta}(\bar{\Omega})$ for some $\beta>0$ and $\Omega$ of class $C^{2, \beta}$.

If $c(x) \equiv c_{0}$ is constant then it is easy to see that $\bar{\lambda}=\underline{\lambda}=c_{0}$ and by Proposition 7.1 the only eigenfunctions are the constants. Nevertheless, if $c(x)$ is not constant the two principal eigenvalues never coincide, unless $\mathcal{M}_{a, A}^{+}$is the Laplacian. To prove this we need the following lemma, whose proof is given for the sake of completness.

Lemma 8.1. Suppose that $\Omega$ is a $C^{2, \beta}$ domain, $b \in C^{0, \beta}(\bar{\Omega})$ and $c \in C^{0, \beta}(\bar{\Omega})$, for some $0<\beta \leq 1$. Then the viscosity solutions of

$$
\begin{cases}-\Delta u+b(x) \cdot D u+c(x) u=0 & \text { in } \Omega \\ \langle D u, \vec{n}(x)\rangle=0 & \text { on } \partial \Omega,\end{cases}
$$

are in $C^{2}(\bar{\Omega})$.

Proof. Consider the problem

$$
\begin{cases}-\Delta v+b(x) \cdot D v+v=f(x) & \text { in } \Omega \\ \langle D v, \vec{n}(x)\rangle=0 & \text { on } \partial \Omega,\end{cases}
$$

where $f(x)=(1-c(x)) u(x)$. By Theorem 5.1, $u$ is Lipschitz continuous on $\bar{\Omega}$ and then the function $f$ is Hölder continuous on $\bar{\Omega}$. Moreover, it is clear that $u$ is a solution of (8.2). The classical theory says that (8.2) has a solution $v \in C^{2}(\bar{\Omega})$. By uniqueness of viscosity solutions of (8.2), we find that $u=v$.

Proposition 8.2. Assume the hypothesis of Lemma 8.1 and let $b \in C^{0,1}(\bar{\Omega})$. If $A \neq a$ and $\bar{\lambda}=\underline{\lambda}$ then $c(x)$ is constant.

Proof. Let $\phi$ be a positive eigenfunction of $\bar{\lambda}$, i.e.

$$
\begin{cases}-\mathcal{M}_{a, A}^{+}\left(D^{2} \phi\right)+b(x) \cdot D \phi+(c(x)-\bar{\lambda}) \phi=0 & \text { in } \Omega \\ \langle D \phi, \vec{n}(x)\rangle=0 & \text { on } \partial \Omega,\end{cases}
$$

and let $-\psi$ be a negative eigenfunction corresponding to $\underline{\lambda}$. Since $\mathcal{M}_{a, A}^{+}\left(-D^{2} \psi\right)=$ $-\mathcal{M}_{a, A}^{-}\left(D^{2} \psi\right), \psi$ satisfies

$$
\begin{cases}-\mathcal{M}_{a, A}^{-}\left(D^{2} \psi\right)+b(x) \cdot D \psi+(c(x)-\underline{\lambda}) \psi=0 & \text { in } \Omega \\ \langle D \psi, \vec{n}(x)\rangle=0 & \text { on } \partial \Omega .\end{cases}
$$

If $\bar{\lambda}=\underline{\lambda}$ then by Proposition $7.1 \psi=t \phi$ for some $t>0$. We can assume $\psi=\phi$. By summing the first equations in (8.3) and (8.4), we can see that $\phi$ is a positive viscosity solution of

$$
\begin{cases}-(A+a) \Delta \phi+2 b(x) \cdot D \phi+2(c(x)-\bar{\lambda}) \phi=0 & \text { in } \Omega \\ \langle D \phi, \vec{n}(x)\rangle=0 & \text { on } \partial \Omega .\end{cases}
$$


Then by Lemma 8.1, $\phi \in C^{2}(\bar{\Omega})$. Subtracting the first equations in (8.3) and (8.4), we can see that $\phi$ is a classical solution of

$$
(A-a) \sum_{i=1}^{N}\left|e_{i}(x)\right|=0 \quad \text { in } \Omega,
$$

where $e_{1}(x), \ldots, e_{N}(x)$ are the eigenvalues of $D^{2} \phi(x)$. Since $A \neq a$, the last equation implies that $e_{i}(x)=0$ in $\Omega$ for any $i=1 \ldots N$. In particular, taking into consideration the boundary condition, $\phi$ is a classical solution of

$$
\begin{cases}\Delta \phi=0 & \text { in } \quad \Omega \\ \langle D \phi, \vec{n}(x)\rangle=0 & \text { on } \quad \partial \Omega,\end{cases}
$$

and then has to be constant. This implies

$$
c(x)-\bar{\lambda}=0 \quad \text { in } \Omega,
$$

i.e., $c \equiv \bar{\lambda}$ is constant.

\section{Acknowledgements}

The author wishes to thank the Professor Hitoshi Ishii for the fruitful discussions about the topics of this paper.

\section{References}

[1] H. Amann, Fixed point equations and nonlinear eigenvalue problems in ordered Banach spaces, SIAM Rev., 18 (1976), 620-709.

[2] A. Anane, Simplicité et isolation de la première valeur propre du p-Laplacien avec poids. (French) [Simplicity and isolation of the first eigenvalue of the p-Laplacian with weight] C. R. Acad. Sci. Paris Sr I Math., 305 (1987), no. 16, 752-728.

[3] M. Bardi and I. Capuzzo Dolcetta, Optimal control and viscosity solutions of Hamilton-Jacobi-Bellmann equations, Birkhäuser, 1997.

[4] G. Barles, Nonlinear Neumann boundary conditions for quasilinear degenerate elliptic equations and applications, J. Differential Equations, 154 (1999), 191-224.

[5] G. Barles and F. Da Lio, Local $C^{0, \alpha}$ estimates for viscosity solutions of Neumanntype boundary value problems, J. Differential Equations, 225 (2006), no. 1, 202-241.

[6] H. Berestycki, L. Nirenberg and S.R.S. Varadhan, The principal eigenvalue and maximum principle for second order elliptic operators in general domain, Comm. Pure Appl. Math., 47 (1994), no. 1, 47-92.

[7] I. Birindelli and F. Demengel, Comparison principle and Liouville type results for singular fully nonlinear operators, Ann. Fac. Sci. Toulouse Math., 13 (2004), no. 2 , 261-287.

[8] I. Birindelli and F. Demengel, Eigenvalue, maximum principle and regularity for fully nonlinear homogeneous operators, Comm. Pure Appl. Anal., 6 (2007), no. 2, 335-366. 
[9] J. Busca, M. J. Esteban, A. Quaas, Nonlinear eigenvalues and bifurcation problems for Pucci's operators, Ann. Inst. H. Poincaré Anal. Non Linéaire, 22 (2005), no. 2, 187-206.

[10] L. Caffarelli and X. Cabré, Fully nonlinear equations. Colloquium Publications 43, American Mathematical Society, Providence, RI, 1995.

[11] M. C. Crandall, H. Ishii and P. L. Lions, User's guide to viscosity solutions of second order partial differential equations, Bull. Amer. Math. Soc. (N.S.), 27 (1992), no. 1, $1-67$.

[12] W. H. Fleming and H. M. Soner, Controlled Markov processes and viscosity solutions. Applications of Mathematics (New York), 25. Springer-Verlag, New York, 1993.

[13] Y. Giga and M. Ohnuma, On strong comparison principle for semicontinouos viscosity solutions of some nonlinear elliptic equations, preprint.

[14] D. Gilbarg and N. S. Trudinger, Elliptic partial differential equations of second order. Reprint of the 1998 edition. Classics in Mathematics. Springer-Verlag, Berlin, 2001.

[15] H. Ishii, Fully nonlinear oblique derivative problems for Nonlinear Second-Order Elliptic PDE's, Duke Math. J., 62 (1991), no. 3, 633-661.

[16] H. Ishii, Perron's method for Hamilton-Jacobi Equations, Duke Math. J., 55 (1987), 369-384

[17] H. Ishii, Personal Communication.

[18] H. Ishii and P. L. Lions, Viscosity Solutions of Fully Nonlinear Second-Order Elliptic Partial Differential Equations, J. Differential Equations, 83 (1990), no. 1, 26-78.

[19] H. Ishii and Y. Yoshimura, Demi-eigenvalues for uniformly elliptic Isaacs operators, preprint.

[20] P. Lindqvist, On a nonlinear eigenvalue problem, Fall. School in Analysis (Jyväskylä, 1994), 33-54. Report, 68, Univ. Jyväskylä, Jyväskylä, 1995.

[21] P. L. Lions, Bifurcation and optimal stochastic control, Nonlinear Anal., 7 (1983), no. $2,177-207$.

[22] E. Milakis and L.E. Silvestre, Regularity for fully nonlinear elliptic equations with Neumann boundary data, Comm. Partial Differential Equations, 31 (2006), no. 7-9, $1227-1252$.

[23] S. Patrizi, The Neumann problem for singular fully nonlinear operators, to appear in Journal de Mathématiques Pure et Appliquées.

[24] M. H. Protter and H.F. Weinberger, Maximum principles in differential equations. Prentice-Hall, Inc., Englewood Cliffs, N.J. 1967.

[25] M. H. Protter and H. F. Weinberger, On the spectrum of general second order operators, Bull. AMS, 72 (1966), 251-255.

[26] A. Quaas, Existence of positive solutions to a "semilinear" equation involving the Pucci's operators in a convex domain, Differential Integral Equations, 17, (2004), no. 5-6, 481-494.

[27] A. Quaas and B. Sirakov, On the principal eigenvalues and the Dirichlet problem for fully nonlinear operators, C. R. Math. Acad. Sci. Paris, 342, no. 2, 115-118. 
Stefania Patrizi

SAPIENZA Università di Roma

Dipartimento di Matematica

Piazzale A. Moro 2

I-00185 Roma

Italy

e-mail: patrizi@mat.uniroma1.it

Received: 24 April 2008.

Accepted: 27 November 2008. 